\title{
Nouvelle vie de couple, nouvelle vie commune? Processus de remise en couple après une séparation
}

Ces dernières décennies, la conjugalité a connu en France d'importantes mutations, dans deux directions au moins. D'une part, les parcours conjugaux se caractérisent désormais par leur discontinuité. Les séparations sont de plus en plus fréquentes et les premières unions durent moins longtemps (12\% des premières unions formées entre 1970 et 1978 étaient rompues dans les huit ans, 29 \% de celles formées entre 1997 et 2005 ; Costemalle, 2015). La remise en couple est devenue un événement banal du parcours de vie ( $\mathrm{Wu}$ et Schimmele, 2005). Moins de cinq ans après une rupture survenue entre 25 et 50 ans, la moitié des personnes cohabitent de nouveau avec un ou une partenaire (Costemalle, 2015). D’une façon générale, les relations amoureuses importantes se multiplient au cours de l'existence (Rault et Régnier-Loilier, 2015).

D'autre part, le couple, autrefois synonyme de mariage, apparaît aujourd'hui protéiforme. En 2011, parmi l'ensemble des personnes vivant en couple (tous âges confondus), $73 \%$ étaient mariées, $23 \%$ vivaient en union libre et $4 \%$ étaient pacsées (Buisson et Lapinte, 2013). Cette statistique transversale rend néanmoins imparfaitement compte de l'ampleur des évolutions récentes. Être en couple sans être marié s'est imposé comme une forme à part entière d'union, au point que la France figure à présent parmi les pays où la cohabitation hors mariage est la plus répandue (Prioux, 2009). Chaque année, le nombre de pacs $^{(1)}$ contractés se rapproche un peu plus du nombre de mariages célébrés (192000 pacs pour 228000 mariages en 2017 ; Papon et Beaumel, 2018). Enfin, près d'un adulte sur dix se déclare en « relation amoureuse stable » sans pour autant habiter avec son ou sa partenaire (Régnier-Loilier et al.,

(1) Le pacte civil de solidarité est un contrat d'union civile créé en 1999 et destiné à la fois aux couples de même sexe et aux couples de sexe différent.

* Institut national d'études démographiques (Ined).

Correspondance : Arnaud Régnier-Loilier, Institut national d'études démographiques, 133, boulevard Davout, 75020 Paris. Courriel : arnaud.regnier-loilier@ined.fr 
$2009)^{(2)}$. Cette situation recouvre toutefois des réalités fort différentes selon le moment où la relation s'inscrit dans le cycle de vie, comme l'a mis en évidence la construction de typologies, tant à partir de données qualitatives (par exemple, Duncan et Philips, 2010; Duncan et al., 2013; Stoilova et al., 2014) que quantitatives (Régnier-Loilier et al., 2009; Reimondos et al., 2011; Pasteels et al., 2015; Coulter et Hu, 2017). Situation contrainte pour certains (en raison de l'éloignement géographique, par exemple), ne pas emménager ensemble représente pour d'autres un choix, en particulier après une séparation.

Ces deux évolutions concomitantes invitent à interroger le lien entre l'histoire conjugale et la forme des unions. Dans la mesure où la configuration conjugale $^{(3)}$ n'est pas fixe, mais où elle évolue au fil de la relation (plus de neuf relations cohabitantes sur dix sont précédées d'une période de non-cohabitation; Costemalle, 2015), il convient d'envisager ce lien dans une perspective dynamique, processuelle. Toutefois, les données disponibles se limitent généralement à la recension des histoires conjugales cohabitantes, conduisant à étudier la remise en couple sous le seul angle de la vie commune. Elles laissent ainsi de côté les relations non cohabitantes et, plus largement, les moments de la relation où chacun a résidé chez soi.

L'enquête Étude des parcours individuels et conjugaux (Épic, Ined-Insee, 2013-2014) a retracé l'ensemble du parcours amoureux et conjugal de chaque répondant, en incluant les périodes de fréquentation où les partenaires ne vivent pas sous le même toit. Cet article, qui se concentre sur la période suivant la dissolution d'un couple cohabitant, étudie la propension à cohabiter ou non dans le cadre d'une nouvelle relation et ses variations en fonction d'un ensemble de caractéristiques.

Certains déterminants connus de la remise en couple cohabitant, ainsi que les résultats d'études portant sur les couples qui ne résident pas ensemble, nous conduisent d'abord à formuler plusieurs hypothèses de recherche. Nous décrivons ensuite la situation conjugale des personnes au moment de l'enquête de façon transversale, avant de nous intéresser, dans une perspective longitudinale, à la probabilité qu'ils cohabitent en fonction de leur histoire conjugale. Enfin, nous modélisons le risque instantané d'emménager ensemble au fil du temps dans le cadre d'une nouvelle relation, selon un ensemble de facteurs : caractéristiques sociales, histoire conjugale et génésique passée des partenaires, caractéristiques de la séparation et de la relation en cours. La période de noncohabitation peut être appréhendée comme un révélateur des manières dont les acteurs s'engagent dans une nouvelle relation et des attentes différenciées qu'ils peuvent nourrir à cet égard.

(2) Lordre de grandeur est similaire dans d'autres pays, comme en Allemagne, en Russie (Liefbroer et al., 2015), en Australie (Reimondos et al., 2011), en Italie (Régnier-Loilier et Vignoli, 2018), au Canada (Turcotte, 2013), en Grande-Bretagne (Haskey, 2005) ou encore aux États-Unis (Strohm et al., 2009).

(3) La « configuration conjugale » renvoie ici à la fois au type d'union (union libre, pacs, mariage) et à la cohabitation (corésidence ou non des partenaires). 


\section{I. Étudier le processus de remise en couple}

\section{Facteurs associés à la formation d'une nouvelle union}

La remise en couple cohabitant a fait l'objet de nombreuses études. Plusieurs tendances ressortent de manière récurrente. En premier lieu, plus l'âge à la séparation est élevé, plus la probabilité de former une nouvelle union est faible, et ce de façon plus prononcée pour les femmes (Cassan et al., 2001; Wu et Schimmele, 2005; Beaujouan, 2009, 2012; Costemalle, 2015; Schimmele et Wu, 2016). En lien avec l'âge, le statut matrimonial joue également. Une étude canadienne montre, notamment, que les veufs et veuves se remettent moins vite en couple que les personnes divorcées, elles-mêmes moins promptes à reformer une union que celles qui n'étaient pas mariées (Wu et Schimmele, 2005). Une partie des personnes divorcées évitent de revivre en couple cohabitant afin de ne plus se retrouver dans une situation de dépendance qu'elles ont pu connaître durant leur mariage (Levin, 2004), tandis que veufs et veuves expriment moins d'intérêt à rencontrer un nouveau partenaire (Carr, 2004). Pour les femmes, le veuvage marque aussi parfois la fin d'une période durant laquelle elles ont tenu un rôle d'aidante auprès de leur mari; après le deuil, elles acquièrent un sentiment d'indépendance et renouent des liens avec leur réseau social (De Jong Gierveld, 2002).

Le fait d'avoir des enfants est également un frein à la formation d'une nouvelle union cohabitante pour les femmes (Bernhardt, 2000), qui s'en voient majoritairement confier la résidence après une séparation. Or la présence de jeunes enfants au domicile limite les possibilités de sorties, donc les opportunités de rencontres (Ivanova et al., 2013; Botterman et al., 2014). Par ailleurs, des conjoints potentiels peuvent être réticents à se mettre en couple avec une femme ayant des enfants à charge, anticipant d'éventuelles difficultés liées à l'exercice de la beau-parentalité (Stewart et al., 2003), tandis que les mères peuvent craindre qu'une nouvelle cohabitation ne s'accompagne de tensions entre leur(s) enfant(s) et le nouveau conjoint (Martin, 1994). À l'inverse, les pères ayant la garde de leurs enfants se remettent généralement plus vite en couple que ceux qui ne l'ont pas (Goldscheider et Sassler, 2006). Lorsque la séparation survient à 45 ans ou après, à des âges où la présence de jeunes enfants au domicile est plus rare, la différence entre les sexes perdure cependant (Schimmele et $\mathrm{Wu}, 2016)$. C'est seulement lorsque la rupture intervient tôt dans le cycle de vie, avant 25 ans ou lorsque les personnes n'ont pas encore d'enfants, que femmes et hommes mettent le même temps à reformer une union (Beaujouan, 2012; Costemalle, 2015).

Si les probabilités de revivre en couple cohabitant sont largement documentées, les études quantitatives laissent le plus souvent dans l'ombre une partie du processus de reconjugalisation ${ }^{(4)}$, entendu ici comme la phase de construction

(4) Les données statistiques ne contiennent généralement aucune information sur la période qui précède un éventuel emménagement du couple, expliquant que la plupart des travaux de recherche se focalisent sur la formation d'une nouvelle union corésidente. On peut néanmoins citer la récente étude allemande d'Ingmar Rapp (2018), qui porte sur la remise en couple au sens large, incluant les relations non corésidentes, réalisée à partir du German Socio-Economic Panel. 
de la relation amoureuse et le temps de fréquentation avant un éventuel emménagement ensemble, dont on peut faire l'hypothèse qu'il varie selon l'histoire conjugale passée. Larticle de Nathalie Beltzer et Michel Bozon (2006) figure parmi les rares études statistiques à sortir du cadre étroit des seules remises en couple cohabitant, en s'intéressant à la vie affective et sexuelle après une rupture conjugale. Les auteurs observent que les femmes connaissent plus souvent que les hommes leur nouveau partenaire au moment de la séparation (sans avoir nécessairement eu de relations sexuelles avec lui avant) et que ce dernier devient plus souvent le nouveau conjoint avec qui elles vont vivre. Pour les hommes, en revanche, le « degré de renouvellement des partenaires » (p. 545) après la séparation est plus important. Beltzer et Bozon montrent également que l'accès à une vie sexuelle post-conjugale est moindre chez les femmes de 35 ans et plus que chez les hommes du même âge et que chez les femmes plus jeunes. Un âge plus élevé correspond à une durée moyenne de vie en couple précédente plus longue, engagement conjugal durable dont on peut faire l'hypothèse qu'il laisserait plus de traces subjectives chez les femmes que chez les hommes et réduirait leurs aspirations à rencontrer un nouveau partenaire. Toutefois, les données sur lesquelles s'appuyait cette étude ${ }^{(5)}$ ne permettaient pas aux auteurs de tenir compte de la situation parentale des personnes au moment de la séparation, ni de s'intéresser à la durée de la fréquentation précohabitationnelle.

Plus récemment, Sofie Vanassche, Martine Corijn et Koen Matthijs (2015b) ont identifié différentes trajectoires conjugales post-divorce en Flandre, parmi lesquelles celle de personnes qui restent durablement en relation non cohabitante (il s'agit d'hommes et de femmes en moyenne plus âgés au moment de la séparation et plus diplômés). Le suivi sur plusieurs années de personnes engagées dans une relation amoureuse stable sans cohabitation a, par ailleurs, montré qu'en France celles qui étaient divorcées entretenaient des relations «chacun chez soi» plus durables que les jeunes, pour qui la non-cohabitation s'apparente à une "période d'essai » menant rapidement à l'emménagement ou à la rupture (Régnier-Loilier, 2016a, 2016b). La non-cohabitation, que les Anglo-Saxons désignent par l'acronyme LAT (Living Apart Together), prend ainsi une signification fort différente selon qu'elle s'inscrit dans un processus de primo-conjugalité ou dans un processus de reconjugalisation, justifiant qu'on ne l'appréhende pas comme un phénomène univoque.

\section{Pistes de recherche}

L'enquête Étude des parcours individuels et conjugaux (Épic), conduite par l'Ined et l'Insee en 2013-2014 en France métropolitaine, retrace l'ensemble du parcours amoureux et conjugal des 26-65 ans de manière détaillée, en distinguant, notamment, le début de la relation et le début de la cohabitation, et fournit un certain nombre de précisions sur la dernière séparation (encadré).

(5) Enquête sur la sexualité "Knowledge, Attitude, Belief and Practice", ORS, 2001. 


\section{Encadré. L'enquête Étude des parcours individuels et conjugaux (Épic)}

L'enquête Épic a été réalisée en population générale en France métropolitaine par l'Ined et I'Insee fin 2013-début 2014 auprès de 7825 femmes et hommes âgés de 26 à 65 ans (pour plus de détails sur l'enquête, voir Rault et Régnier-Loilier, 2019, dans ce numéro de Population). La question suivante leur était posée afin de connaître leur situation conjugale au moment de l'enquête : "Êtes-vous actuellement en couple? Oui, avec une personne qui vit dans le logement/Oui, avec une personne qui ne vit pas dans le logement/Non ».

L'ensemble des " relations de couple ou relations amoureuses importantes » (sans aucune restriction de durée ni critère de cohabitation) était retracé. II s'agissait notamment de décrire différentes étapes de chaque relation : date de début, dates éventuelles de début de cohabitation, de mariage, de pacs, de naissance des enfants, de fin de relation, de décohabitation, de divorce ou de rupture du pacs. On connaît ainsi l'âge du répondant au début de chacune de ses relations, sa situation conjugale (avoir été ou non marié/pacsé) et parentale (avoir déjà des enfants, leur âge, vivre ou non avec eux), la durée de chaque relation ou encore le temps écoulé entre chacune d'elles.

En outre, la dernière séparation d'une union cohabitante a donné lieu à une description détaillée. Celle-ci intègre notamment quelques questions visant à saisir le ressenti des répondants : «Cette séparation a-t-elle été déstabilisante pour vous sur le plan affectif? Oui beaucoup/Oui un peu/Non », "Et du point de vue matériel et financier? ». On sait également si les ex-conjoints ont « continué à vivre sous le même toit après avoir décidé de se séparer » et leur entente (« Au moment de la séparation, diriez-vous que vos relations avec [Prénom] étaient.... Excellentes/Bonnes/Ni bonnes, ni mauvaises/Conflictuelles, tendues/Violentes /Inexistantes ou presque »).

Quelques informations relatives au passé conjugal des différents partenaires du répondant sont renseignées : " [Prénom] avait-il/elle déjà vécu en couple? », " Avait-il/elle été marié(e)? ", " [Prénom] avait-il/elle eu des enfants de son côté avant le début de votre relation? », « [Prénom] vivait-il/elle avec ses enfants (nés d'une autre relation)? ".

Épic offre donc deux perspectives d'analyse. De manière transversale (partie II), elle permet d'étudier la situation conjugale au moment de l'enquête en distinguant les personnes en couple cohabitant, celles en couple non cohabitant et celles qui ne se déclarent pas en couple; et, parmi ces dernières, celles qui sont en « relation amoureuse importante » (non cohabitante) et celles qui n'ont pas de relation. De manière longitudinale (partie III), l'enquête permet d'étudier différentes séquences du processus de formation d'une nouvelle relation et, notamment, le temps écoulé entre le début de la relation en cours et l'éventuel emménagement dans un logement commun.

Elle permet ainsi l'étude des dynamiques de remise en couple cohabitant après une rupture, à l'aune des caractéristiques du passé conjugal. Le regard croisé des travaux portant sur la remise en couple, d'une part, et sur les LAT, d'autre part, suggère quatre hypothèses principales.

Premièrement, on peut s'attendre à ce que le temps passé en non-cohabitation soit plus important pour les femmes lorsqu'elles ont des enfants à charge, la résidence de ces derniers leur étant confiée bien plus fréquemment ${ }^{(6)}$ qu'aux

(6) Un an après un divorce, 76 \% des enfants mineurs sont gardés principalement par leur mère (Bonnet et al., 2015). 
hommes. Cette situation s'accompagne d'une probabilité de se remettre en couple cohabitant plus faible chez les mères (Beaujouan, 2012; Vanassche et al., 2015a). Claude Martin (1994) note par ailleurs qu'elles optent plus souvent pour une vie de couple non cohabitant. La plupart des études qualitatives sur les LAT mettent en avant le « choix » des mères de ne pas cohabiter avec leur nouveau partenaire afin d'éviter de perturber leurs enfants en leur imposant une figure beau-parentale (Bawin-Legros et Gauthier, 2001; Levin, 2004; Duncan et al., 2013). En revanche, les comportements de remise en couple cohabitant des parents d'enfants majeurs ne différant guère de ceux des personnes sans enfant (Vanassche et al., 2015a), on s'attend à ce que cette configuration parentale ne joue pas sur le temps passé en non-cohabitation.

Deuxièmement, on avance l'hypothèse qu'une relation conjugale antérieure de longue durée, marquée par un mariage (donc généralement suivie d'un divorce), dont la séparation a été déstabilisante sur le plan affectif ou matériel, peut conduire à une attitude de prudence dans le cadre d'une nouvelle relation. Cela contribuerait à retarder l'emménagement dans un logement commun, le temps de s'assurer de la solidité de la relation en conservant un lieu de repli (Villeneuve-Gokalp, 1997), voire à réduire le souhait de s'installer de nouveau en couple et risquer de revivre une séparation qui a pu laisser des souvenirs douloureux (Levin, 2004; Duncan et al., 2013).

Troisièmement, et en lien avec l'hypothèse précédente, on peut envisager l'après-séparation comme une période de reconstruction de soi qui prend plus ou moins de temps, une période durant laquelle on (ré)apprend à vivre seul(e), où l'on accède à une certaine forme de liberté et où le réseau des relations amicales s'élargit (Villeneuve-Gokalp, 1994). On peut alors supposer que les personnes ont d'autant plus de mal à renoncer à cette liberté (re)trouvée en s'installant avec quelqu'un qu'elles ont mis de temps à s'engager dans une nouvelle relation (temps écoulé entre la séparation et le début de la relation en cours). La noncohabitation permettrait ainsi une mise à distance de la vie conjugale commune pour continuer à se définir personnellement, par soi-même (Charrier, 2008).

Quatrièmement, l'hypothèse d'un processus d'installation sous le même toit plus rapide pour les personnes se situant en bas de la hiérarchie sociale peut être formulée, pour deux raisons. Tout d'abord, les contraintes économiques plus fortes peuvent pousser à faire résidence commune plus vite afin de réaliser certaines économies d'échelle offertes par la cohabitation -un seul loyer au lieu de deux, etc. ${ }^{(7)}$ - (Haskey et Lewis, 2006). Corrélativement, on peut s'attendre à une plus forte propension à vivre en couple sans cohabiter en haut de l'échelle sociale. $\mathrm{Si}$, contrairement à ce qui s'observait dans les anciennes générations, le niveau de diplôme ne constitue plus un élément distinctif du statut matrimonial (Prioux,

(7) Une hypothèse concurrente pourrait cependant être avancée : si l'emménagement dans un logement commun risque de conduire à la perte de certaines aides sociales, cela pourrait inciter les partenaires à rester dans une relation non cohabitante (De Jong Gierveld, 2002; Connidis et al., 2017). De même, l'instabilité professionnelle, plus fréquente en bas de l'échelle des diplômes, pourrait augmenter la probabilité d'être non cohabitant (Castro-Martín et al., 2008). 
2009), la préférence pour telle ou telle forme d'union varie selon le milieu social. Par exemple, Estelle Bailly et Wilfried Rault (2013) mettent en évidence que les personnes qui optent pour le pacs sont tendanciellement plus diplômées que celles qui choisissent de se marier. Par ailleurs, Aart Liefbroer et al. (2015) trouvent en Europe une légère corrélation positive entre le niveau de diplôme et la probabilité d'être en couple non cohabitant. Cela pourrait traduire des conceptions socialement différenciées de la vie à deux, avec, pour les classes sociales les moins élevées, une moindre ouverture aux formes alternatives au couple " classique », tandis que les plus diplômées résisteraient davantage aux normes familiales traditionnelles et pourraient peut-être devenir les précurseurs d'un nouveau mode de conjugalité, chacun chez soi (Liefbroer et al., 2015).

Afin d'éprouver ces hypothèses, il est procédé dans un premier temps à une analyse descriptive, transversale d'abord, longitudinale ensuite, à partir de fonctions de survie (Kaplan-Meier), puis à une analyse par modélisation (modèle de Cox) permettant de mesurer l'effet propre de différents facteurs sur le risque instantané de cohabiter au fil de la relation plutôt que de rester en couple non cohabitant.

\section{Champ de l'étude}

Cette étude porte sur les personnes ayant déjà vécu en couple cohabitant par le passé (pour lesquelles on dispose d'un ensemble d'informations sur la dernière séparation) ${ }^{(8)}$ et qui, au moment de l'enquête, sont engagées dans une nouvelle relation de couple ou relation amoureuse importante (encadré), cohabitante ou non (configurations $a, b$ et $c$, figure 1). L'objectif est d'analyser la durée de la période de non-cohabitation des relations en cours au moment de l'enquête (représentée dans la figure par la lettre A) en fonction des caractéristiques de la relation cohabitante qui a précédé et des modalités de la séparation $^{(9)}$. Dans la configuration $a$, soit la relation en cours est cohabitante et a éventuellement été précédée d'une période de non-cohabitation, soit elle est toujours non cohabitante à la date de l'enquête (censure à droite, qui sera prise en compte dans les analyses). Dans la configuration $b$, peu fréquente, la relation en cours a débuté alors que le ou la répondant.e résidait encore avec son exconjoint.e. Dans un quart des cas, toutefois, cette précédente relation avait pris fin, même si la cohabitation se poursuivait (le temps de s'organiser, etc.); dans les autres cas, la période de chevauchement entre les deux relations a été le plus souvent brève (moins de huit mois, dans la moitié des cas). Dans les configurations $c$ et $d$, une ou plusieurs relations non corésidentes ont suivi la dernière relation cohabitante ${ }^{(10)}$ mais étaient terminées à la date de l'enquête. Létude se concentrant sur les relations en cours au moment de l'enquête, la

(8) Entente du couple au moment de la séparation; avoir continué, ou non, à cohabiter une fois prise la décision de se séparer; conséquences affectives et économiques de la séparation.

(9) Seules les modalités de la dernière séparation qui a marqué la fin d'une union cohabitante sont décrites dans l'enquête.

(10) Elle a pu commencer, dans quelques cas, avant la séparation. 
configuration $d$, qui n'en comprend pas, n'est donc pas analysée ici (hors champ $)^{(11)}$. Dans la configuration $c$, seule la durée de non-cohabitation de la relation en cours est étudiée. Pour autant, l'occurrence d'une relation non cohabitante entre la fin de la relation cohabitante rompue et le couple actuel sera prise en compte dans les analyses.

Ces choix méthodologiques appellent certaines précisions en matière de représentativité. L'étude concerne des unions en cours au moment de l'enquête et n'est donc pas représentative des personnes séparées. Les personnes seules à l'enquête ont pu connaître un épisode amoureux sans cohabitation (configuration d), d'autres engageront une relation dans l'avenir. Par ailleurs, l'orientation sexuelle n'est pas prise en compte dans l'analyse. Bien que femmes et hommes en couple de même sexe soient proportionnellement plus nombreux à être en couple avec quelqu'un qui réside dans un autre logement (Buisson et Lapinte, 2013; RégnierLoilier, 2018), les effectifs ne sont pas suffisants dans Épic pour étudier la spécificité de leurs comportements dans l'après-séparation (19 hommes et 18 femmes). Par ailleurs, les personnes dont la dernière relation cohabitante a pris fin en raison du décès du ou de la conjoint(e), trop peu nombreuses dans l'enquête pour permettre une exploitation spécifique, ne sont pas incluses dans l'étude.

Figure 1. Cas de figure présents dans les données Épic et durée étudiée (A)

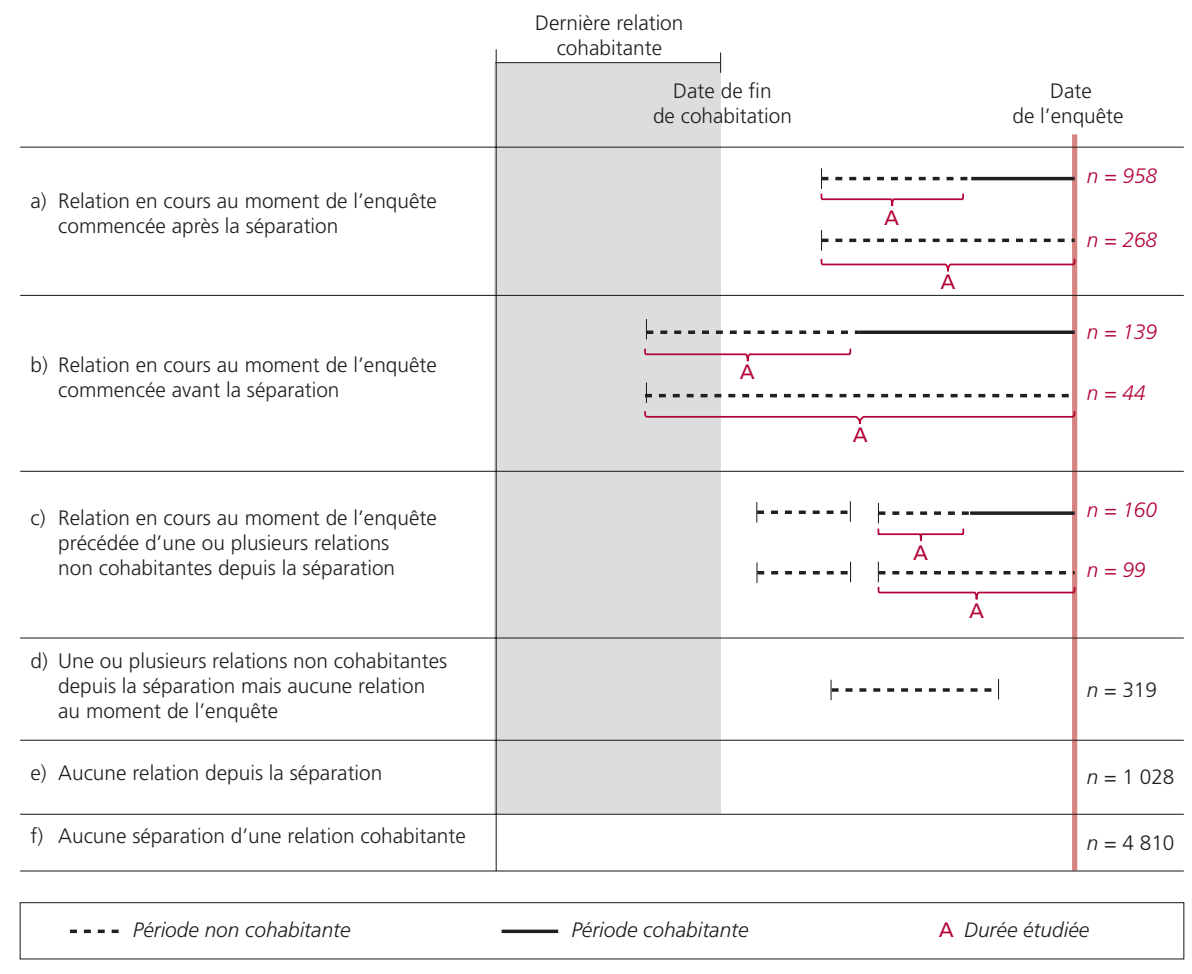

(11) Notons que, par construction, s'ils avaient cohabité, cette période n'aurait pas été étudiée : elle aurait correspondu à la « dernière relation cohabitante". 


\section{Repérer les couples non cohabitants}

\section{Une configuration difficile à identifier avec précision dans les enquêtes}

Jusqu'à récemment, les enquêtes ne collectaient le plus souvent que les situations de couple cohabitant. Les personnes qui ne vivaient pas avec un.e conjoint.e étaient parfois interrogées sur l'existence d'une éventuelle relation amoureuse stable avec quelqu'un résidant ailleurs (comme dans l'enquête Étude des relations familiales et intergénérationnelles) ${ }^{(12)}$. Près de $10 \%$ des adultes (18-79 ans) se reconnaissaient dans ce cas en France en 2005, mais cette notion de « relation amoureuse stable » englobe une grande variété de situations (RégnierLoilier et al., 2009) et seul un tiers d'entre eux décrivaient leur relation « plutôt comme une relation de couple " (Régnier-Loilier, 2018). Ce n'est qu'à partir de 2011, en France, avec l'enquête Famille et logements (EFL), qu'une question a été introduite afin de repérer directement les «couples» non cohabitants ${ }^{(13)}$ : « Êtes-vous actuellement en couple? Oui, avec une personne qui vit dans le logement; oui, avec une personne qui vit dans un autre logement; non, mais vous avez déjà été en couple par le passé; non, vous n’avez jamais été en couple » ${ }^{(14)}$. D’après l'EFL, seuls $3 \%$ des adultes (18-79 ans) se déclarent en couple avec un conjoint qui ne vit pas dans leur logement (Régnier-Loilier, 2018).

Dans l'enquête Épic, sur laquelle s'appuie cet article, la même question a été posée au tout début du questionnaire, afin que la réponse ne soit pas influencée par d'autres questions de l'enquête. En particulier, il était demandé plus loin aux personnes interrogées de décrire l'ensemble de leurs « relations de couple ou relations amoureuses importantes $»$. Cette définition extensive des relations aurait pu inciter davantage de personnes à se déclarer en couple « avec une personne qui ne vit pas dans le logement». Malgré cette double précaution (formulation et position dans le questionnaire), la comparaison des deux sources met en lumière d'importants écarts de fréquence à tout âge (figure 2). En moyenne, dans la tranche d'âges commune aux deux enquêtes (26-65 ans), la proportion de personnes se déclarant en couple avec quelqu'un vivant dans un autre logement est près de trois fois supérieure dans Épic (un peu moins de $6 \%$ contre $2 \%$ dans EFL).

Au moins deux raisons sont susceptibles d'expliquer ces écarts. Premièrement, la thématique générale de l'enquête, clairement annoncée aux enquêté.e.s dans

(12) Enquête Érfi (Ined-Insee, 2005-2008-2011), version française du programme Generations and Gender Survey.

(13) Auparavant, l'enquête « Situations familiales » (Ined-Insee, 1985) avait intégré dans la description de l'histoire matrimoniale la question suivante : "Aviez-vous une résidence commune ou bien avez-vous toujours conservé deux résidences ", question qui, toutefois, n'était posée que pour les périodes « de vie en couple». On peut supposer que les périodes où les personnes étaient en couple sans partager le même logement n'ont pas toujours été déclarées comme des périodes de vie en couple mais ont pu être déclarées comme des périodes de vie seule.

(14) Enquête réalisée par l'Insee auprès de 359669 femmes et hommes âgés de 18 ans ou plus. 
Épic comme portant sur les parcours conjugaux, a pu inciter davantage de personnes ne vivant pas en couple cohabitant mais entretenant une relation amoureuse à se reconnaître dans la catégorie « en couple avec une personne qui ne vit pas dans le logement». Deuxièmement, le mode de collecte a pu jouer un rôle. Alors que le questionnaire d'EFL était auto-administré (sans enquêteur), les questions d'Épic étaient posées par une enquêtrice ou un enquêteur, qui a pu apporter des précisions sur ce que l'on entendait dans l'enquête par « être en couple ». Même si aucune définition précise n'était donnée dans les "instructions de collecte ", enquêtrices et enquêteurs étaient néanmoins sensibilisé.e.s à l'acception large visée par Épic. À l'inverse, le questionnaire d'EFL venait en complément de celui du recensement de la population, dans lequel il était demandé aux personnes interrogées si elles « vivaient » en couple. Par souci de cohérence, il est possible que certaines, ayant répondu dans le recensement ne pas "vivre en couple », aient également déclaré ne pas «être en couple » dans EFL. Quelles qu'en soient les raisons, ces différences illustrent combien il est difficile de repérer précisément les « couples non cohabitants ». Alors que le mariage ou la cohabitation constituent des événements assez clairement identifiables, la frontière entre le couple et le hors couple dans le cadre de relations non cohabitantes est moins évidente et laissée à l'appréciation de chacun, donc sujette à d'importantes variations.

Figure 2. Proportion de femmes et $d$ 'hommes en couple non cohabitant par âge

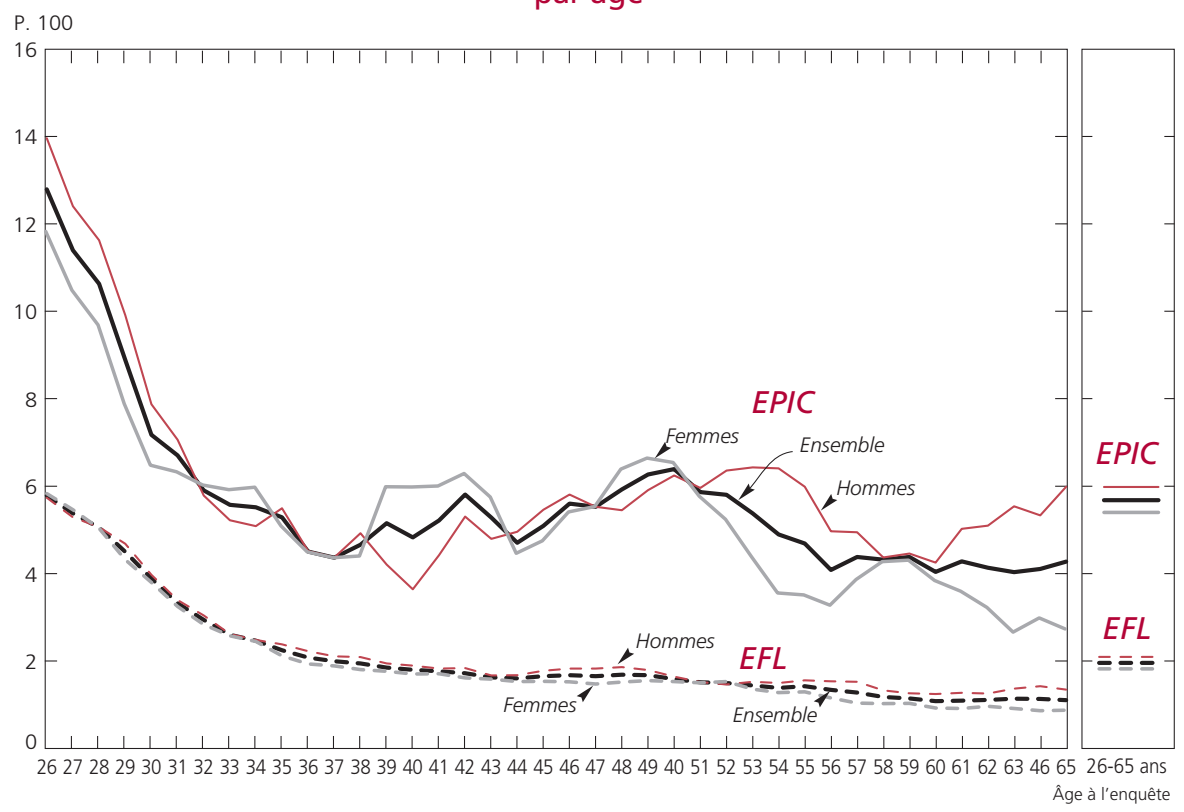

Note : Moyennes mobiles d'ordre 5 (la proportion à un âge a correspond à la moyenne pondérée des proportions observées aux âges $a-2, a-1, a, a+1, a+2)$.

Champ : 26-65 ans (France métropolitaine, logements ordinaires).

Sources : EFL (Insee, 2011); Épic (Ined-Insee, 2013-2014). 


\section{La non-cohabitation, plus fréquente en deuxième partie de vie amoureuse}

Quelle que soit la source retenue, on observe une part plus importante de personnes se disant en couple avec quelqu'un qui réside dans un autre logement aux plus jeunes âges (figure 2). À ces âges, la non-cohabitation correspond souvent à une première relation de couple et s'apparente à une étape transitoire, qui conduit assez rapidement à un emménagement dans un logement commun ou à une rupture (Régnier-Loilier, 2016a). La non-cohabitation se fait ensuite plus rare, au profit de la conjugalité cohabitante. Le léger regain observé entre 40 et 55 ans dans Épic - mais non visible dans EFL- pourrait correspondre à des situations post-séparation. Après une rupture, s'ouvre une période de remise en couple. Et, comme mentionné précédemment, la probabilité de remise en union cohabitante décline avec la durée de l'union précédente et dépend, notamment, de la situation parentale au moment de la séparation. Or, à ces âges, la présence d'enfants est fréquente et peut conduire à s'engager dans une relation sans pour autant emménager avec son ou sa nouvelle partenaire.

La situation de couple (cohabitant ou non cohabitant) des personnes au moment de l'enquête Épic apparaît ainsi étroitement liée à leur histoire amoureuse et conjugale (figure 3). Quatre situations sont distinguées dans Épic : les

Figure 3. Situation de couple au moment de l'enquête selon l'histoire amoureuse et conjugale, et le sexe

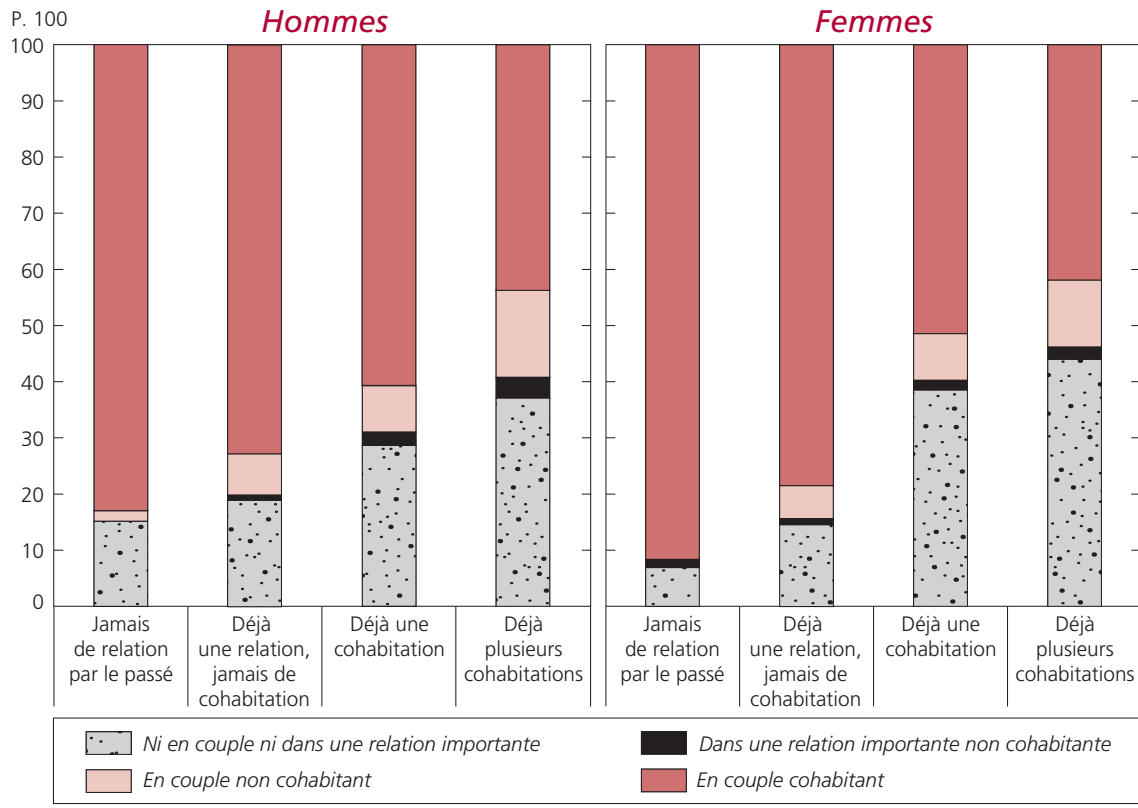

Lecture: Au moment de l'enquête, 83 \% des hommes n'ayant jamais eu de relation amoureuse importante par le passé sont en couple cohabitant.

Champ : 26 à 65 ans (France métropolitaine, logements ordinaires).

Source : Épic (Ined-Insee, 2013-2014). 
personnes en couple cohabitant, celles en couple avec quelqu'un qui réside ailleurs, celles qui ne se considèrent pas en couple mais déclarent une « relation amoureuse importante " (non cohabitante) et celles qui ne sont pas engagées dans une relation.

La proportion de personnes hors couple (ni en couple ni dans une relation amoureuse importante) au moment de l'enquête est d'autant plus élevée qu'elles ont eu des relations auparavant : $30 \%$ des hommes et $39 \%$ des femmes ayant déjà vécu en couple cohabitant se trouvent dans cette situation contre $15 \%$ des hommes et $7 \%$ des femmes n'ayant pas connu de relation amoureuse importante précédemment. De la même manière, la part de personnes en « couple non cohabitant » est nettement plus élevée parmi celles ayant déjà vécu en couple cohabitant ( $10 \%$ environ après une première cohabitation) que parmi celles n'ayant jamais eu de relation avant celle en cours lors de l'enquête (moins de $2 \%$ ).

Ces différences peuvent tenir à deux effets conjugués : d'une part, après la séparation, les personnes peuvent opter pour une forme de couple dans lequel chacun garde son propre logement (attitude de prudence avant de revivre avec quelqu'un, par exemple); d'autre part, l'après-séparation peut conduire à des situations qui freinent l'installation sous le même toit (présence d'enfants, par exemple).

\section{Analyser la dynamique de formation d'une nouvelle union à l'aune de l'expérience conjugale}

\section{Propension à vivre ensemble selon l'âge et I'histoire amoureuse : approche descriptive}

Intéressons-nous à présent plus spécifiquement à la dynamique de formation d'un nouveau couple après une rupture, en privilégiant l'approche longitudinale. Les différences observées en coupe transversale laissent supposer que le passé conjugal joue sur le calendrier d'emménagement, voire sur le choix de la forme conjugale (en corésidence ou chacun chez soi). Afin d'étudier de manière dynamique le temps passé en non-cohabitation en fonction de l'histoire passée, tout en tenant compte du fait que certaines personnes n'ont pas encore emménagé avec leur partenaire mais le feront peut-être plus tard (censure à droite), nous avons utilisé une fonction de survie (méthode de Kaplan-Meier), qui mesure, à chaque mois d'ancienneté de la relation en cours, la probabilité d'être toujours non cohabitant (figure 4) ${ }^{(15)}$. Aucune différence significative n'ayant été relevée entre femmes et hommes, les deux sexes sont ici regroupés.

(15) La fenêtre d'observation est limitée à 96 mois : au-delà de huit ans, la survenue d'événements devient marginale. 
Figure 4. Probabilité de rester en couple non cohabitant selon I'histoire conjugale (courbe de survie Kaplan-Meier)

Probabilité de rester en couple non cohabitant

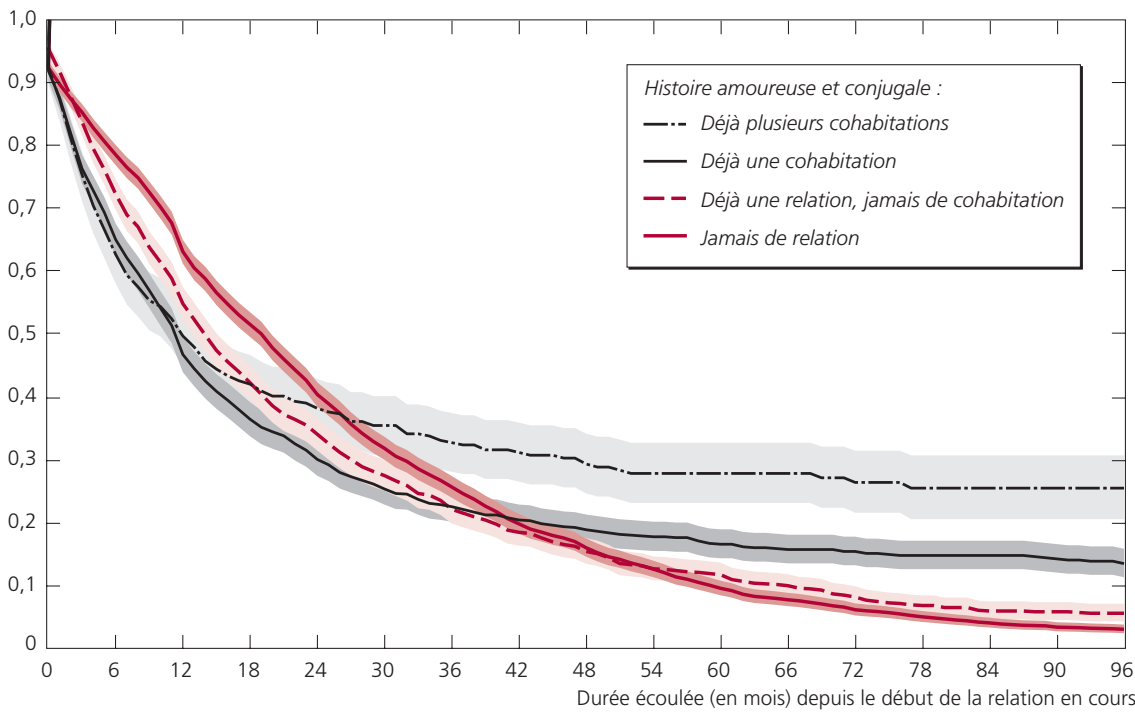

Lecture : 18 mois après le début de la relation, $51 \%$ des personnes n'ayant jamais eu de relations avant I'union en cours, sont toujours non cohabitantes.

Note : Les plages colorées représentent les intervalles de confiance à $95 \%$.

Champ : 26-65 ans en couple ou relation amoureuse importante (France métropolitaine, logements ordinaires)

Source : Épic (Ined-Insee, 2013-2014)

De manière générale, lorsqu'une relation dure, elle évolue assez rapidement vers une installation sous le même toit. Après 15 mois, la probabilité d'être toujours non cohabitant est inférieure à $50 \%$; au bout de deux ans, elle n'est plus que d'un tiers. L'expérience conjugale joue de manière contrastée sur le calendrier d'emménagement. Bien que les personnes n'ayant connu aucune expérience amoureuse importante auparavant soient proportionnellement très peu nombreuses à être en couple non cohabitant au moment de l'enquête (2\%), leur probabilité d'emménager dans les premiers mois de la relation est la moins élevée. Après un an et demi de relation, la moitié d'entre elles sont encore en relation non cohabitante contre un tiers de celles ayant déjà vécu une fois en couple cohabitant.

Cela tient en partie à un effet d'âge au début de la relation (ce que confirme la figure 5) et s'explique par la situation dans laquelle se trouvent alors les personnes. Les plus jeunes, n'ayant aucune expérience passée, sont pour certaines encore en études au moment de la rencontre, situation peu compatible avec la cohabitation. Ressources limitées et incertitudes quant au lieu de résidence futur (qui se précisera au moment de l'entrée dans la vie active) conduisent les jeunes à repousser l'installation en couple à plus tard (Giraud, 2017). Par ailleurs, les jeunes adultes ne veulent pas devenir « adultes » trop 
tôt (Singly, 2000) en s'enfermant dans une relation cohabitante pendant que leurs ami.e.s du même âge « sortent en discothèque » (Giraud, 2017). En revanche, au bout de cinq ans (60 mois), la probabilité d'être toujours non cohabitant dans le cadre d'une première relation amoureuse est inférieure à $10 \%$, contre $17 \%$ si la personne a déjà vécu une fois en couple cohabitant et 27 \% après au moins deux périodes de cohabitation (figure 4). L'expérience d'une cohabitation passée conduit donc moins souvent à l'installation sous le même toit.

Figure 5. Probabilité de rester en couple non cohabitant selon l'âge au début de la relation (courbe de survie Kaplan-Meier)

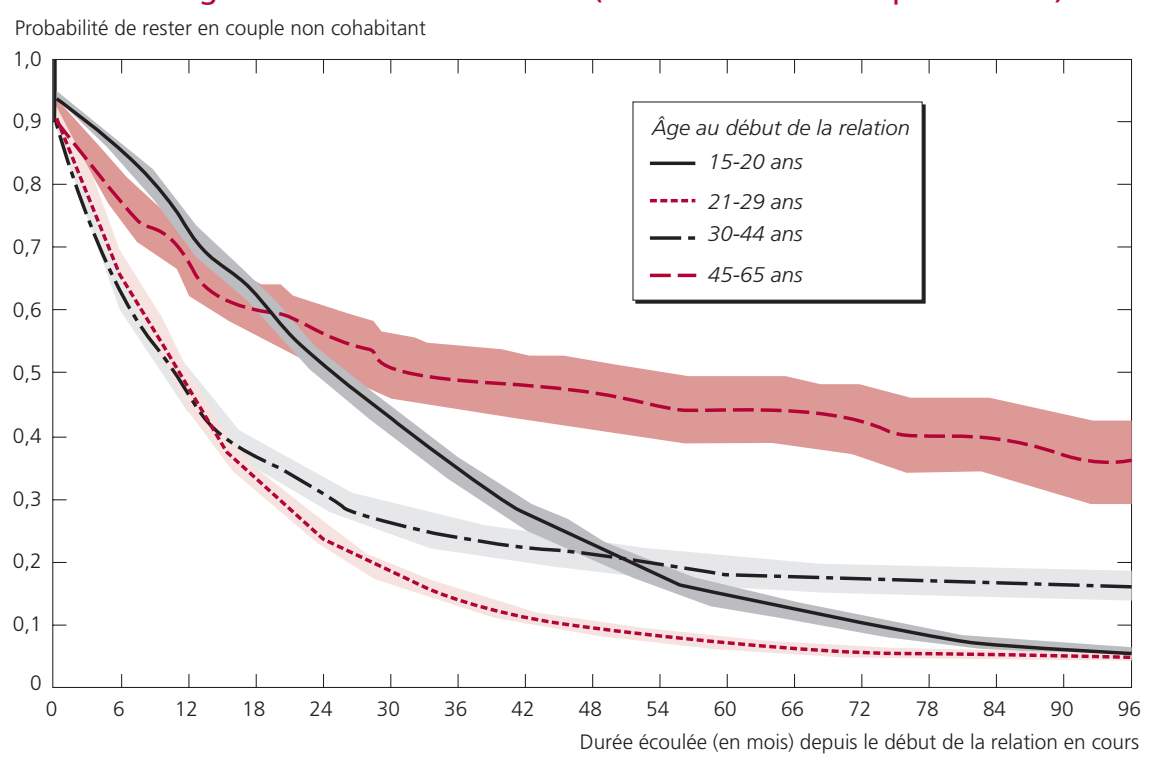

Lecture et note : cf. figure 4.

Champ : 26-65 ans en couple ou relation amoureuse importante (France métropolitaine, logements ordinaires).

Source : Épic (Ined-Insee, 2013-2014).

Deux logiques différentes sont ainsi mises au jour avec, d'un côté, des personnes jeunes (moins de 30 ans), qui n'ont jamais vécu en couple cohabitant auparavant et pour qui la probabilité d'être toujours dans une relation non cohabitante est négligeable au bout de huit ans, et, de l'autre, des personnes ayant déjà vécu en couple, pour qui l'emménagement à terme est moins probable. Ces résultats corroborent ceux obtenus à partir de données de panel, montrant une faible survie en non-cohabitation pour les plus jeunes, pour qui la relation chacun chez soi est une période de test qui conduit rapidement (après quelques années) soit à la séparation, soit à l'emménagement, tandis qu'elle est davantage inscrite dans la durée pour les personnes ayant déjà vécu en couple (Régnier-Loilier, 2016a). C'est sur cette population spécifique des personnes ayant déjà cohabité que porte la suite de l'analyse, avec pour objectif d'étudier l'effet des caractéristiques de l'histoire conjugale sur le processus de remise en couple. 


\section{Dynamique de formation}

\section{d'une nouvelle union selon l'expérience conjugale}

L'histoire conjugale ne se résume pas au nombre d'expériences passées. $\mathrm{Au} v u$ des études s'intéressant aux remises en couple cohabitant, on peut s'attendre à ce que l'expérience matrimoniale (avoir été marié ou non, durée de la relation) et parentale (avoir eu ou non des enfants, leur âge et lieu de résidence) antérieure de chacun des partenaires influe sur le processus de remise en couple et la forme de la nouvelle union. En outre, d'autres facteurs peuvent jouer, comme le sexe, le milieu social, lâge au début de la relation en cours au moment de l'enquête, la stabilité de cette dernière, le temps écoulé depuis la dernière relation cohabitante, les expériences amoureuses survenues dans l'intervalle ou encore les modalités de la séparation (qualité des relations avec l'ex-partenaire au moment de la séparation, avoir continué à cohabiter une fois prise la décision de se séparer, rupture déstabilisante du point de vue matériel ou affectif) ${ }^{(16)}$.

Certaines de ces caractéristiques sont en partie liées entre elles. Par exemple, les unions longues ont aussi plus souvent donné lieu à un mariage et à l'arrivée d'enfants. Afin de tester nos hypothèses, nous recourons à un modèle semi-paramétrique à risques proportionnels (modèle de Cox) visant à faire ressortir l'effet net de chaque facteur sur le processus de reconjugalisation. En d'autres termes, il s'agit d'estimer le risque instantané d'avoir emménagé, sous la forme d'une fonction dépendante du temps (durée écoulée depuis le début de la relation en cours, en mois), pour les personnes ayant déjà vécu en couple cohabitant par le passé et engagées dans une relation, cohabitante ou non, au moment de l'enquête.

Trois modèles incluant l'ensemble des variables listées ci-dessus sont présentés (tableau 1$)^{(17)}$ : un premier regroupant les deux sexes, puis un modèle pour chaque sexe. Les comportements sexuels et conjugaux des femmes et des hommes après une séparation étant différents (Beltzer et Bozon, 2008), on souhaite ainsi voir si des spécificités selon le sexe apparaissent dans le processus de formation d'une nouvelle union.

\section{La propension à cohabiter varie peu entre femmes et hommes}

Le premier modèle («Ensemble », tableau 1) ne laisse guère entrevoir de différence significative selon le sexe quant au risque instantané d'emménager avec le ou la nouvelle partenaire dans le cadre d'une nouvelle relation. Ce résultat pourrait, à première vue, paraître contradictoire au regard des études réalisées

(16) Voir encadré pour la formulation des questions.

(17) L'hypothèse de proportionnalité des risques (visant à mesurer la constance des rapports de risque sur l'ensemble de la durée d'observation) a été testée pour chaque variable, en deux temps. Un premier test a été réalisé sur les résidus standardisés de Schoenfeld puis, pour les quelques variables qui pouvaient laisser suspecter un non-respect de l'hypothèse, une interaction linéaire avec la durée a été introduite. Seul l'âge au début de la relation (modalité " avant 30 ans ») a laissé apparaître une interaction significative, qui est donc conservée dans les modèles. 
Tableau 1. Risque instantané de cohabiter avec son nouveau partenaire vs d'être toujours non cohabitant (rapports de risques (RR), modèle de Cox)

\begin{tabular}{|c|c|c|c|c|c|c|c|}
\hline & \multicolumn{2}{|c|}{ Ensemble } & \multicolumn{2}{|c|}{ Hommes } & \multicolumn{3}{|c|}{ Femmes } \\
\hline & $\mathrm{RR}$ & $\mathrm{n}$ & RR & $\mathrm{n}$ & RR & & $n$ \\
\hline \multicolumn{8}{|c|}{ Caractéristiques sociodémographiques du/de la répondant·e } \\
\hline \multicolumn{8}{|c|}{ Sexe } \\
\hline Homme (Réf.) & 1 & 728 & & & & & \\
\hline Femme & 0,98 & 940 & & & & & \\
\hline \multicolumn{8}{|l|}{ Niveau de diplôme } \\
\hline Aucun diplôme, CEP, BEPC & $1,29 * *$ & 285 & 1,25 & 115 & 1,43 & $\star \star *$ & 170 \\
\hline CAP, BEP & 1,16 & 452 & 1,19 & 221 & 1,23 & * & 231 \\
\hline Bac (Réf.) & 1 & 289 & 1 & 130 & 1 & & 159 \\
\hline BTS, DEUG, licence & 1,09 & 452 & 1,24 & 173 & 1,05 & & 279 \\
\hline Diplôme supérieur & 0,89 & 190 & 1,01 & 89 & 0,79 & & 101 \\
\hline \multicolumn{8}{|c|}{ Histoire conjugale et familiale de chaque partenaire } \\
\hline Histoire matrimoniale du/de la répondant.e & & & & & & & \\
\hline N’a jamais été marié·e (Réf.) & 1 & 888 & 1 & 401 & 1 & & 487 \\
\hline A déjà été marié·e & $1,23 * \star *$ & 780 & $1,40 * * *$ & 327 & 1,11 & & 453 \\
\hline \multicolumn{8}{|l|}{$\begin{array}{l}\text { Situation parentale du/de la répondant.e } \\
\text { au début de la relation }\end{array}$} \\
\hline Sans enfant (Réf.) & 1 & 756 & 1 & 347 & 1 & & 409 \\
\hline Enfant.s majeur.s vivant ailleurs & 0,91 & 164 & 1,11 & 81 & 0,75 & & 83 \\
\hline Enfant.s mineur.s vivant ailleurs & 0,88 & 494 & 1,01 & 250 & 0,77 & $\star \star$ & 244 \\
\hline Enfant $\cdot s$ cohabitant $\cdot s$ & $0,69 * * *$ & 254 & 0,83 & 50 & 0,61 & $\star \star \star$ & 204 \\
\hline \multicolumn{8}{|l|}{ Histoire matrimoniale dulde la partenaire } \\
\hline N’a jamais vécu en couple cohabitant & 0,95 & 467 & 0,88 & 193 & 1,00 & & 274 \\
\hline N’a jamais été marié·e (Réf.) & 1 & 566 & 1 & 274 & 1 & & 292 \\
\hline A déjà été marié·e & 0,97 & 635 & $0,75 * *$ & 261 & 1,16 & & 374 \\
\hline \multicolumn{8}{|l|}{$\begin{array}{l}\text { Situation familiale du/de la partenaire } \\
\text { au début de la relation }\end{array}$} \\
\hline Sans enfant (Réf.) & 1 & 900 & 1 & 395 & 1 & & 505 \\
\hline Enfant.s vivant ailleurs & $0,73 * * *$ & 586 & 0,82 & 227 & 0,69 & $\star \star \star$ & 359 \\
\hline Enfant $\cdot s$ cohabitant $\cdot s$ & 0,82 & 182 & 0,77 & 106 & 0,90 & & 76 \\
\hline \multicolumn{8}{|l|}{$\begin{array}{l}\text { Durée de la relation précédente } \\
\text { (cohabitation) }\end{array}$} \\
\hline Moins de 5 ans & 1,06 & 705 & 1,02 & 323 & 1,08 & & 382 \\
\hline De 5 ans à moins de 10 ans (Réf.) & 1 & 416 & 1 & 173 & 1 & & 243 \\
\hline 10 ans et plus & 0,97 & 547 & 1,08 & 232 & 0,84 & & 315 \\
\hline \multicolumn{8}{|l|}{ Caractéristiques de la séparation } \\
\hline \multicolumn{8}{|l|}{$\begin{array}{l}\text { Qualité des relations avec l'ex-partenaire } \\
\text { au moment de la séparation }\end{array}$} \\
\hline Bonnes & 0,96 & 266 & 1,03 & 133 & 0,90 & & 133 \\
\hline $\begin{array}{l}\text { Ni bonnes, ni mauvaises; } \\
\text { inexistantes (Réf.) }\end{array}$ & 1 & 387 & 1 & 200 & 1 & & 187 \\
\hline Conflituelles, tendues, violentes & 0,91 & 1015 & 1,00 & 395 & 0,83 & $\star *$ & 620 \\
\hline \multicolumn{8}{|l|}{$\begin{array}{l}\text { Poursuite de la cohabitation } \\
\text { après avoir décidé de se séparer }\end{array}$} \\
\hline Non (Réf.) & 1 & 1163 & 1 & 544 & 1 & & 619 \\
\hline Oui & $0,86 * *$ & 505 & $0,80 * *$ & 184 & 0,93 & & 321 \\
\hline \multicolumn{8}{|l|}{$\begin{array}{l}\text { Séparation déstabilisante } \\
\text { du point de vue matériel }\end{array}$} \\
\hline Non (Réf.) & 1 & 1257 & 1,00 & 583 & 1 & & 674 \\
\hline Oui & 0,95 & 411 & 1,05 & 145 & 0,90 & & 266 \\
\hline \multicolumn{8}{|l|}{$\begin{array}{l}\text { Séparation déstabilisante } \\
\text { du point de vue affectif }\end{array}$} \\
\hline Non (Réf.) & 1 & 896 & 1 & 425 & 1 & & 471 \\
\hline Oui & 0,98 & 772 & 0,94 & 303 & 1,02 & & 469 \\
\hline
\end{tabular}


Tableau 1 (suite). Risque instantané de cohabiter avec son nouveau partenaire vs d'être toujours non cohabitant (rapports de risques, modèle de Cox)

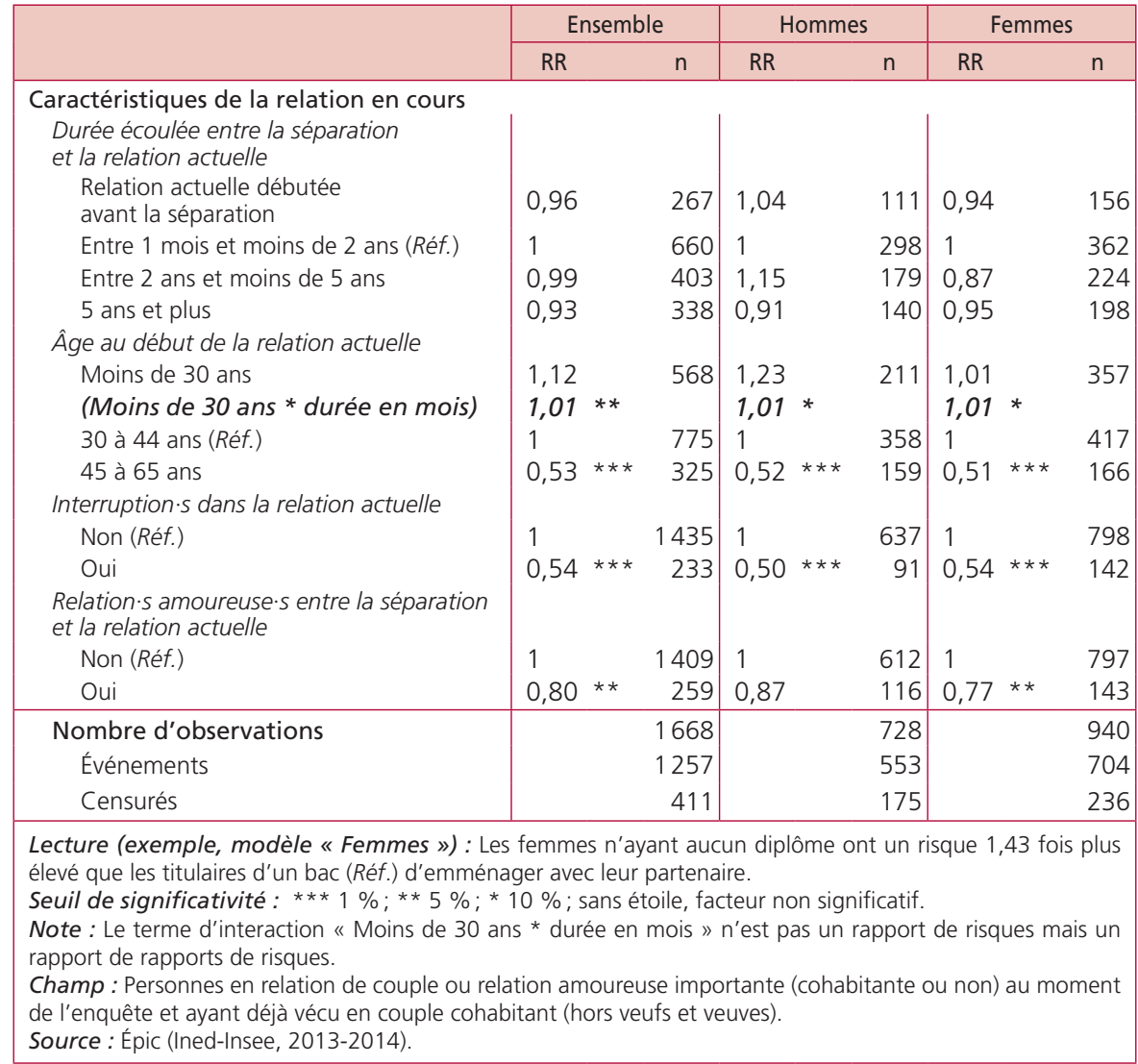

sur la remise en couple, la plupart relevant une moindre probabilité des femmes à reformer une union cohabitante. Mais il découle de l'approche différente adoptée ici : l'étude porte sur une population sélectionnée, celles des personnes déjà engagées dans une nouvelle relation (cohabitante ou non). Cette absence d'écart entre sexes suggère que ce n'est pas tant sur le choix de cohabiter ou non, ni le temps passé en non-cohabitation, que se jouent les différences entre sexes, mais plutôt sur la formation même d'une nouvelle relation. Nathalie Beltzer et Michel Bozon (2008) observent qu'après une séparation, les femmes de 35 ans et plus ont moins de chances de rencontrer un nouveau partenaire. En complément de la modélisation, la figure 6 représente la probabilité de ne pas s'engager dans une nouvelle relation (cohabitante ou non) en fonction du temps, selon le sexe. À partir d'Épic, on retrouve bien une tendance moindre des femmes séparées à reformer un couple. Par exemple, au bout de 60 mois, soit cinq ans après la séparation, $55 \%$ des femmes ne sont pas engagées dans une nouvelle relation amoureuse contre $48 \%$ des hommes. Ces différences entre femmes et hommes 
demeurent après contrôle d'autres caractéristiques habituellement retenues dans les études sur le sujet (résultats non présentés ici) ${ }^{(18)}$.

Figure 6. Probabilité de ne pas s'engager dans une nouvelle relation après une séparation, selon le sexe du répondant (courbe de survie Kaplan-Meier)

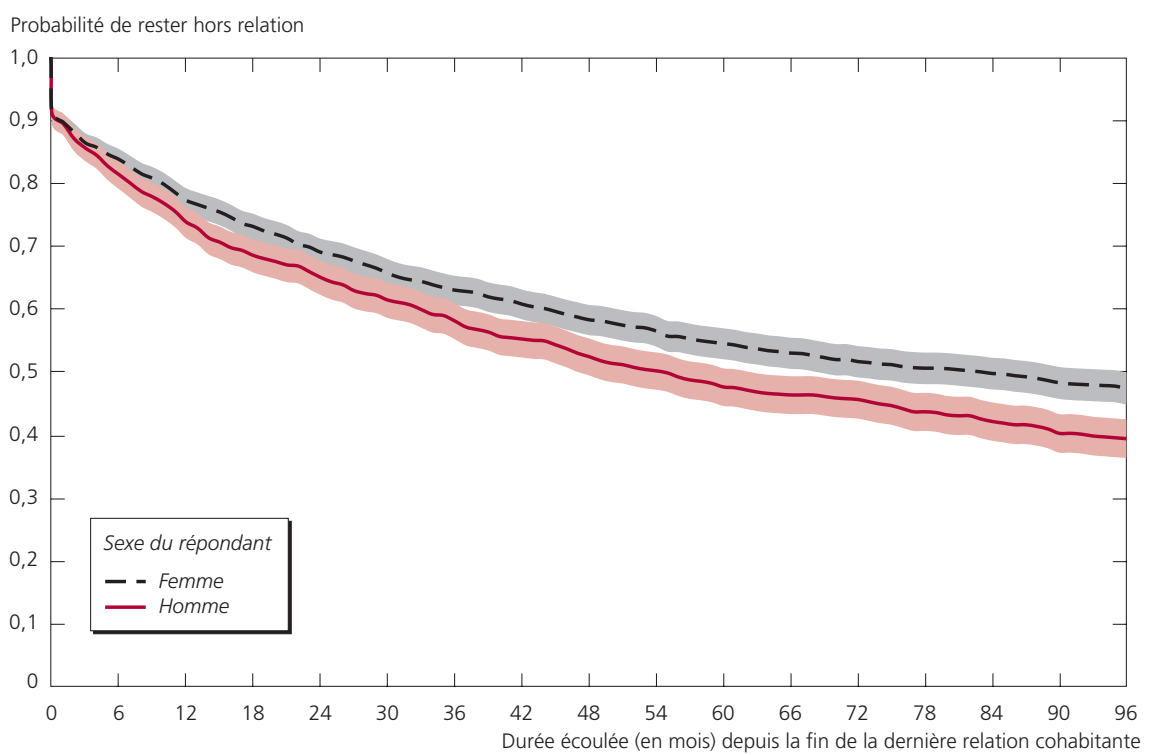

Lecture : 18 mois après une séparation, $73 \%$ des femmes ne sont pas engagées dans une nouvelle relation.

Note : Le décrochage observé à la durée 0 correspond aux personnes engagées dans une nouvelle relation dès leur séparation.

Champ : 26-65 ans ayant déjà vécu en couple cohabitant par le passé (France métropolitaine, logements ordinaires).

Source : Épic (Ined-Insee, 2013-2014).

Comme le suggèrent la plupart de ces études, la différence entre femmes et hommes en matière de calendrier et d'intensité de l'engagement dans une nouvelle relation importante (cohabitante ou non) tient principalement à la présence d'enfants issus d'une précédente union. Elle n'apparaît pas chez les personnes n'ayant pas eu d'enfants, alors que la propension des femmes à reformer un couple est inférieure à celle des hommes lorsque l'union précédente a été féconde (figure 7). Là aussi, la différence est confirmée « toutes choses égales par ailleurs ${ }^{(19)}$. C'est donc avant tout la propension à rencontrer un nouveau partenaire et à s'engager dans une relation amoureuse qui différencie

(18) Variables de contrôle : âge à la séparation, présence d'enfants issus de l'union précédente, statut matrimonial passé, durée de la relation précédente, niveau de diplôme, avoir ou non continué à vivre sous le même toit une fois prise la décision de se séparer, conséquences de la séparation du point de vue matériel, affectif et rang de l'union rompue.

(19) Même modèle que celui décrit précédemment mais incluant une interaction entre le sexe et la présence d'enfants (effet négatif et significatif au seuil de $1 \%$ pour les femmes), et modèle stratifié selon la présence d'enfants (effet négatif et significatif au seuil de $1 \%$ pour les femmes) ou l'absence d'enfants (pas de différence significative entre hommes et femmes). 
les femmes et les hommes à la suite d'une union féconde, plus que la forme que prend ensuite cette relation (entre cohabitation et non-cohabitation).

Figure 7. Probabilité de ne pas s'engager dans une nouvelle relation après une séparation, selon le sexe et la présence d'enfants (courbe de survie Kaplan-Meier)

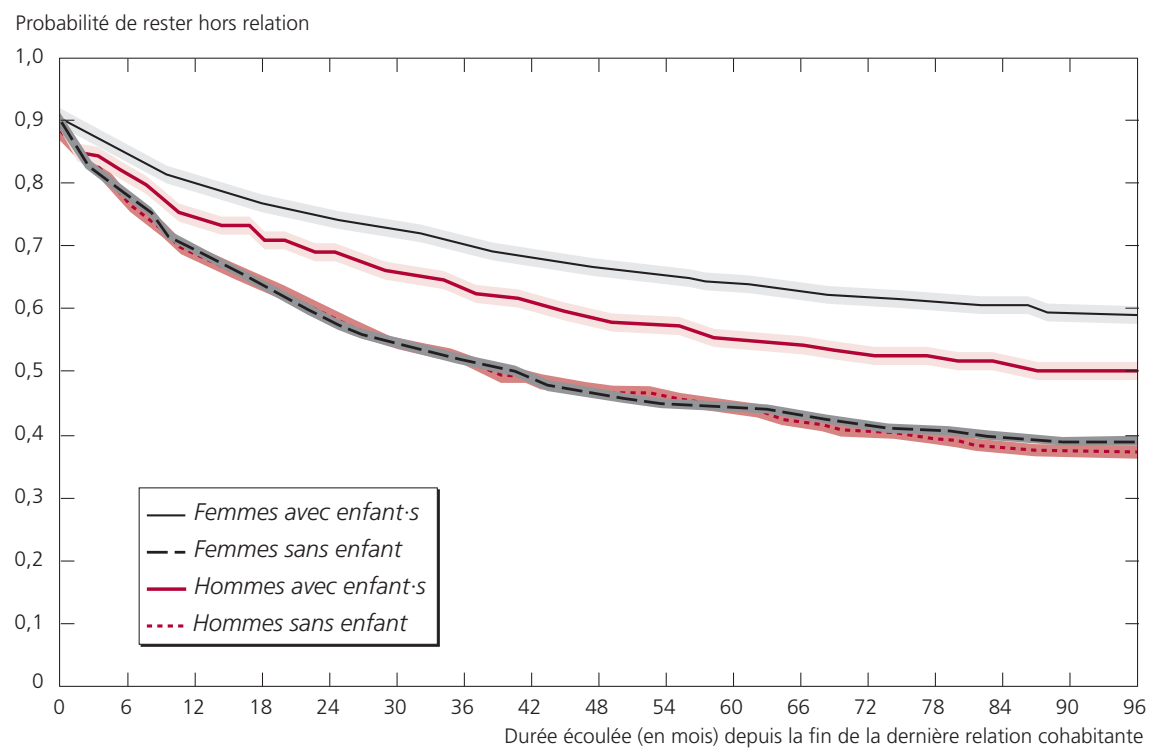

Lecture: $\mathrm{Cf}$. figure 4

Champ : 26-65 ans ayant déjà vécu en couple cohabitant par le passé (France métropolitaine, logements ordinaires).

Source : Épic (Ined-Insee, 2013-2014).

\section{La présence d'enfants réduit, pour les femmes,} la probabilité d'une nouvelle vie commune

Parmi les personnes engagées dans une nouvelle relation, un effet propre de la situation parentale au moment de la remise en couple est mis en évidence du côté des femmes (tableau 1) : le fait d'avoir un ou des enfants mineurs, cohabitant en permanence ou non, réduit le risque instantané de s'installer avec leur nouveau partenaire. L'emménagement est également moins probable lorsque le partenaire est lui-même déjà parent ${ }^{(20)}$. Du côté des hommes, la situation parentale ne fait apparaître aucun effet significatif sur le risque instantané d'emménager dans le cadre d'une nouvelle relation. Cela confirme que l'histoire parentale des hommes pèse moins sur la suite de leur parcours conjugal ou peut traduire des mécanismes différents, mais produisant un même effet. D’un côté, les pères qui ne vivent pas avec leurs enfants seraient plus « libres »

(20) La situation où le père réside avec ses enfants, peu fréquente, a un effet non significatif mais négatif. Notons que cet effet devient significatif dès lors que l'on ne tient pas compte de la situation familiale de la femme (modèle non présenté). 
pour reformer un nouveau couple; de l'autre, ceux qui résident avec eux trouveraient dans la vie commune un soutien à l'exercice de leur parentalité ou seraient des hommes davantage orientés vers la vie familiale, pouvant expliquer qu'aucune différence n'apparaisse entre ces deux cas de figure. La situation parentale de leur nouvelle partenaire n'a pas un effet très clair : si cette dernière réside avec des enfants, le risque instantané d'emménager est moindre, mais non significatif (il le devient néanmoins dès lors que l'on ne prend pas en compte dans le modèle la situation familiale du répondant ${ }^{(21)}$, à l'instar de ce que l'on observe pour les femmes).

En revanche, pour les femmes comme pour les hommes, le fait d'avoir des enfants majeurs vivant ailleurs ne change pas la probabilité de s'installer sous le même toit, toutes choses égales par ailleurs. Dans cette configuration, les partenaires pourraient estimer que les enfants sont moins susceptibles d'être perturbés par une remise en couple cohabitant et/ou que les relations beauxparentales ont moins de risques de troubler la nouvelle relation amoureuse. L'hypothèse selon laquelle le temps passé en non-cohabitation est plus important lorsque la femme a des enfants à charge est ainsi vérifiée.

\section{Avoir été marié joue de manière opposée selon le sexe}

L'effet du statut matrimonial antérieur sur le temps passé dans une nouvelle relation sans habiter avec son ou sa partenaire est en partie confirmé, mais complexe à isoler précisément. En premier lieu, on n'observe pas de symétrie dans les résultats entre les modèles par sexe (tableau 1). Alors que, dans le modèle "Hommes", le risque instantané d'emménager est moindre pour les hommes dont la partenaire a déjà été mariée, aucun effet significatif du mariage de la femme ne ressort dans le modèle «Femmes». Cela peut tenir à la prise en compte de davantage de caractéristiques au niveau " répondant » qu'au niveau « partenaire ». L'histoire conjugale antérieure du ou de la partenaire se limite en effet à un seul indicateur (avoir été ou non marié), alors qu'un ensemble d'autres variables sont prises en compte pour la personne qui répond à l'enquête (durée de l'union passée, contexte et conséquences de la séparation) ${ }^{(22)}$.

Chez les hommes, le fait d'avoir déjà été marié produit un effet inverse à celui observé chez les femmes, favorisant pour eux la vie commune dans le cadre d'une nouvelle relation, à situation parentale, durée de relation passée ou encore âge au début de la relation donnés. Ce résultat est cohérent avec l'étude de Francine Cassan et al. (2001), qui a mis en évidence une plus forte probabilité des divorcés à revivre en couple cohabitant. Cela pourrait être lié à un effet de sélection des personnes ayant déjà été mariées, le choix du mariage pouvant aller de pair avec une préférence ou un attrait pour la vie de couple, les poussant à reformer plus volontiers une nouvelle union (Bernhardt, 2000).

(21) Modèle non présenté.

(22) Dans une enquête, il n'est en effet pas possible de demander à un répondant de décrire la relation passée et le contexte de séparation qu’a vécu son ou sa partenaire. 
Aucun effet significatif de la durée de la relation passée n'est observé une fois prises en compte les autres caractéristiques, en particulier la situation matrimoniale passée ${ }^{(23)}$. Une analyse stratifiée selon le fait d'avoir été ou non marié permet cependant de mettre en évidence que la durée de la relation passée n'a pas d'effet significatif chez les personnes qui étaient mariées, tandis qu'elle est négativement corrélée au risque d'emménager sous le même toit chez celles qui ne l'étaient pas ${ }^{(24)}$. Dit autrement, l'expérience du mariage prime sur la durée de la relation passée et, en l'absence de mariage, celle-ci affecte négativement la remise en couple cohabitant.

Concernant l'expérience de la séparation, la perception de conséquences négatives (déstabilisation sur le plan matériel ou affectif) n'entraîne aucun effet, ni pour les femmes ni pour les hommes. Deux facteurs influent toutefois négativement sur le risque de cohabiter dans le cadre d'une nouvelle relation. Les femmes qui décrivent des relations « conflictuelles, tendues, violentes » avec leur ancien conjoint au moment de la rupture sont moins enclines à emménager avec leur nouveau partenaire. Pour les hommes, le fait d'avoir continué à résider avec leur ex-partenaire, une fois prise la décision de se séparer, joue négativement, cette situation pouvant révéler des difficultés à faire le deuil de la relation passée, marquer une hésitation à se séparer ou encore des difficultés financières et matérielles à surmonter avant de pouvoir cesser la cohabitation.

En définitive, l'effet des caractéristiques de la relation passée (durée, statut matrimonial) et de la séparation va globalement dans le sens attendu, pour les femmes : mariage passé, relation précédente longue, relations conflictuelles avec l'ex-partenaire au moment de la séparation paraissent s'accompagner d'un engagement plus timide vers la cohabitation dans le cadre d'une nouvelle relation. Pour les hommes, seul le fait d'avoir continué à habiter avec l'ex-partenaire après avoir décidé de se séparer réduit le risque d'emménager dans le cadre d'une nouvelle union, tandis qu'avoir déjà été marié est associé à l'effet inverse.

\section{La durée écoulée entre la séparation et la nouvelle relation n'a pas d'effet}

La durée écoulée entre la séparation et le début de la relation en cours au moment de l'enquête ne joue pas sur la propension à emménager avec son ou sa nouvelle partenaire. L'hypothèse d'une probabilité moindre de s'installer sous le même toit lorsque l'on a mis plus de temps à s'engager dans une nouvelle relation n'est donc pas vérifiée (tableau 1, caractéristiques de la relation en cours).

De même, femmes et hommes ayant commencé leur nouvelle relation avant de se séparer de leur ex-partenaire (ou immédiatement après leur séparation)

(23) Ces caractéristiques sont liées entre elles : la durée moyenne de la relation passée est bien plus longue chez les personnes ayant été mariées (12 ans) que chez celles ne l'ayant pas été (5 ans).

(24) Ce résultat est déduit d'une interaction entre la durée de la relation passée et la situation matrimoniale passée significative au seuil de $5 \%$ pour les relations passées ayant duré au moins 10 ans (modèle non présenté). 
n'ont pas un risque instantané de cohabiter significativement différent des autres. Notons que, dans ce type de situation (début de relation actuelle antérieur à la rupture de l'union précédente : figure lb), nous avons considéré que les personnes étaient exposées au risque d'emménager dès le début de leur relation, même si elles résidaient encore avec leur ex-partenaire. En prenant comme point de départ la séparation de l'union antérieure (et non le début de la relation en cours), le risque d'emménager est alors significativement plus élevé pour celles et ceux dont les histoires se sont chevauchées (résultat non présenté) ${ }^{(25)}$. Dans ces cas de figure, l'emménagement est donc plus rapide à partir du moment où la séparation a lieu mais, en définitive, la durée totale passée en non-cohabitation ne diffère pas par rapport aux relations qui se sont succédées sans recouvrement.

\section{Les moins diplômés ont un risque accru de cohabiter}

Un effet marqué du niveau de diplôme (lequel est corrélé au milieu social) ressort chez les femmes, confirmant notre quatrième hypothèse. Le risque instantané d'emménager ensemble est significativement plus élevé chez les moins diplômées et, réciproquement, la tendance s'inverse pour les diplômées de l'enseignement supérieur. L'interprétation de ce résultat reste néanmoins ouverte, plusieurs éléments pouvant jouer. Un haut niveau de qualification s'accompagne d'un niveau de vie moyen plus élevé et donc d'une possibilité accrue d'indépendance résidentielle, avec une moindre pression économique à s'installer ensemble; les personnes les plus diplômées seraient moins sensibles à l'économie d'échelle potentielle que peut représenter le fait de vivre ensemble plutôt que dans des logements séparés ${ }^{(26)}$. Une autre explication renvoie à des conceptions socialement différenciées de la vie de couple, avec un attrait plus prononcé pour la vie commune en bas de l'échelle sociale et, inversement, un penchant plus fort pour l'indépendance résidentielle chez les plus diplômés.

\section{Le risque de vivre ensemble décroît avec l'âge}

Pour finir, quelques caractéristiques de la relation en cours influent sur la propension à vivre ensemble. Premièrement, l'âge au début de la relation joue significativement. Pour les personnes qui se sont réengagées dans une relation avant 30 ans, l'effet n'est pas significatif, comparativement aux 30-44 ans, mais le facteur d'interaction avec la variable dépendante (durée de remise en couple) l'est et est positif. Cela indique un risque croissant de s'installer sous le même toit à mesure que la relation s'installe dans la durée pour les plus jeunes. En revanche, celles et ceux qui étaient âgés de 45 à 65 ans au début de leur nouvelle relation tendent moins à faire résidence commune.

(25) L'hypothèse de proportionnalité dans le modèle de Cox n'est alors pas vérifiée pour cette modalité, réduisant la qualité du modèle.

(26) Bien que le niveau de revenu ne soit connu qu'au moment de l'enquête, nous en avons testé l'effet. La même tendance se dessine, les femmes aux plus bas revenus ayant un risque instantané d'emménager plus fort. 
Deuxièmement, le fait d'être engagé dans une relation ayant été interrompue à une ou plusieurs reprises (sans que l'on connaisse les raisons ni la durée de ces pauses) ${ }^{(27)}$ réduit la probabilité d'emménager ensemble rapidement. Cela peut être le signe de relations plus instables, et donc s'accompagner d'un moindre empressement à partager le même logement. L'effet est aussi « mécanique » : l'interruption momentanée de la relation allonge d'autant la durée considérée ici comme faisant partie de la période de relation non cohabitante ${ }^{(28)}$.

Enfin, avoir vécu une autre relation amoureuse non cohabitante entre sa dernière vie de couple cohabitante et la relation en cours (figure 1c) atténue la propension à cohabiter. Cela pourrait refléter un moindre attrait pour la cohabitation après une séparation. De même, cet épisode de non-cohabitation qui n'a pas abouti à une vie commune pourrait avoir été ressenti comme un nouvel échec et conduire à une certaine prudence avant de s'installer de nouveau dans une relation cohabitante.

\section{Conclusion}

La plupart des enquêtes démographiques et des données disponibles ne permettent pas d'étudier la remise en couple après une séparation autrement qu'en s'intéressant à la formation d'une nouvelle union corésidente. Toutefois, la conjugalité ne se réduit pas à la seule vie commune, en particulier après une séparation. La conjugalité non cohabitante est fréquente dans ce cas. En coupe transversale, près d'un cinquième (18 \%) des femmes et des hommes engagés dans une relation amoureuse après avoir déjà vécu en couple cohabitant ne résident pas avec leur nouveau partenaire. Cette « photographie » souffre cependant de certaines limites, notamment dans le repérage précis des personnes en couple avec quelqu'un vivant dans un autre logement. Sans gommer l'ensemble des difficultés liées à l'identification des épisodes de non-cohabitation, Épic offre la possibilité de distinguer différents moments de la formation d'une relation. Elle permet d'appréhender la remise en couple, non pas comme un état, mais comme un processus, en étudiant plus spécifiquement la durée de la période non cohabitante et ses variations en fonction des situations individuelles. En réponse aux hypothèses formulées, quatre principaux résultats émergent.

Premièrement, une fois qu'ils sont engagés dans une nouvelle relation de couple, l'installation sous le même toit est, en moyenne, aussi rapide et fréquente pour les femmes que pour les hommes. Si la plupart des études montrent que les premières reforment moins souvent une union cohabitante, cela semble lié principalement au fait qu'elles s'engagent moins souvent dans une nouvelle

(27) L'information est issue de la question : «Vous est-il arrivé d'interrompre votre relation avec [Prénom] avant de la reprendre? Oui une fois/Oui, plusieurs fois/Non ».

(28) Il conviendrait en toute rigueur de soustraire le temps de la ou des interruptions de la relation, mais cette information n'est pas disponible; le fait d'avoir connu ou non une pause dans la relation est ainsi pris en compte à titre de contrôle. 
relation amoureuse. Ce n'est pas la vie commune en tant que telle qui serait moins attrayante ou possible pour elles, mais plutôt les occasions de rencontres ou l'envie de s'engager dans une nouvelle relation amoureuse qui seraient moindres.

Deuxièmement, comme de nombreuses études qualitatives l'ont mis en avant, il paraît important de prendre en compte le parcours conjugal antérieur dans létude du processus de remise en couple, en particulier pour les femmes. Avoir été mariées ou avoir connu une relation longue et avoir vécu des relations conflictuelles ou tendues au moment de la séparation réduit leur propension à emménager avec leur nouveau partenaire.

Troisièmement, lâge au début de la nouvelle relation et la situation parentale s'avèrent déterminants quant à la forme que prend la nouvelle union. Plus les personnes sont âgées et moins elles ont tendance à s'engager dans une relation cohabitante. De même, la présence de jeunes enfants (mineurs et/ou corésidents au moment de la séparation) issus d'une précédente union limite la cohabitation, résultat observable uniquement chez les femmes.

Quatrièmement, si la remise en couple cohabitant des femmes et des hommes est moins rapide dans les classes sociales les moins favorisées (Beaujouan, 2009), l'effet du milieu social s'inverse une fois une nouvelle relation engagée. La propension à prendre un logement commun décroît à mesure que l'on grimpe dans l'échelle des diplômes (et des revenus) et inversement, avec un effet plus net chez les femmes. Cela pourrait tenir aux économies d'échelle que peut représenter le fait de vivre à deux ou à des aspirations socialement différenciées vis-à-vis de la vie de couple.

Si le couple non cohabitant est parfois envisagé comme la conséquence possible du développement des valeurs individualistes et d'autonomie propres aux sociétés modernes, cette piste mérite d'être nuancée en l'état actuel des connaissances. S'il est vrai que les personnes en couple non cohabitant développent un discours davantage orienté sur les notions d'épanouissement personnel, d'indépendance et d'autonomie dans leur relation, cette forme d'union reste aujourd'hui peu répandue (entre $2 \%$ et $6 \%$ des 26-65 ans en France, selon la source retenue, figure 2) et s'avère spécifique de certaines périodes du cycle de vie. Aux plus jeunes âges, lorsqu'il s'agit d'une première relation amoureuse importante, elle s'apparente principalement à une étape transitoire liée aux conditions du moment (études en cours, absence d'emploi pour l'un des deux partenaires, relation trop récente), mais la probabilité d'être toujours en couple non cohabitant devient insignifiante après quelques années. Si l'on peut voir dans le couple non cohabitant des signes de la « seconde transition démographique », notamment caractérisée par un individualisme croissant, c'est donc principalement en deuxième partie de vie amoureuse, après avoir expérimenté la vie de couple dans sa forme « classique » (entendue ici comme cohabitante) et notamment après avoir eu des enfants. La non-cohabitation relève alors de différentes logiques, non exclusives les unes des autres : attitude de prudence à l'égard d'un nouvel engagement conjugal, manière de concilier 
de nouvelles aspirations personnelles, préserver ses enfants issus d'une précédente relation et/ou son nouveau partenaire de possibles difficultés liées à la beau-parentalité. Par ailleurs, à ce moment du cycle de vie, les injonctions à cohabiter sont moindres. D'une part, les partenaires ont, de manière générale, moins fréquemment le projet d'avoir des enfants (certains ont déjà ceux qu'ils souhaitaient, voire ne sont plus en âge d'en avoir). Dans un contexte où la vie commune fait partie des conditions préalables à l'arrivée d'un enfant, l'absence de projet parental limite donc l'injonction à vivre ensemble. D'autre part, contrairement aux plus jeunes, les partenaires ont, dans certains cas, déjà leur propre logement, voire en sont propriétaires, ce qui est susceptible de réduire la pression économique à vivre ensemble.

Remerciements : L'auteur tient à remercier Marie Bergström, Wilfried Rault, Marc Thévenin, Sandra Zilloniz pour leur relecture et précieux conseils, ainsi que les évaluateurs anonymes et le comité de rédaction pour leurs suggestions.

L'enquête Épic a été financée par l'Ined, l'Insee, l'Agence nationale de la recherche (ANR, projet CECHIC, "Corpus pour l'étude de cent ans d'histoire du couple en France »), la Caisse nationale des allocations familiales (CNAF), la Direction de la recherche, des études, de l'évaluation et des statistiques (Drees) et le Laboratoire d'excellence iPOPs (individus, populations, sociétés). 


\section{RÉFÉRENCES}

BAILly E., RAUlT W., 2013, Les pacsés en couple hétérosexuel sont-ils différents des mariés ?, Population et sociétés, $n^{\circ} 497$.

BAWIN-Legros B., GAUTHIER A., 2001, Regulation of intimacy and love semantics in couple living apart together, International Review of Sociology, 11(1), p. 39-46.

BEAUjOUAN É., 2009, Se remettre en couple : contrastes hommes-femmes, in Pailhé A., Solaz A. (dir.), Entre famille et travail. Des arrangements de couples aux pratiques des employeurs, Paris, Éditions de l'Ined-La Découverte, p. 259-284.

BEAUJOUAN É., 2012, Repartnering in France: The role of gender, age and past fertility, Advances in Life Course Research, 17(2), p. 69-80.

BELTZER N., BOZON M., 2006, La vie sexuelle après une rupture conjugale. Les femmes et la contrainte de l'âge, Population, 61(4), p. 535-551.

BELTZER N., BOZON M., 2008, Les séparations et leurs suites : rencontres sexuelles et prévention après une rupture conjugale ou amoureuse, in Bajos N., Bozon M. (dir.), Enquête sur la sexualité en France. Pratiques, genre et santé, Paris, La Découverte, p. 197-212.

BERNHARDT E., 2000, Repartnering among Swedish men and women: A case study of emerging patterns in the second demographic transition, Présenté à la FFS Flagship Conference, Bruxelles.

Bonnet C., GARBinti B., SOlaZ A., 2015, Les conditions de vie des enfants après le divorce, Insee première, $\mathrm{n}^{\circ} 1536$.

Botterman S., Sodermans A. K., Matthijs K., 2014, The social life of divorced parents. Do custody arrangements make a difference in divorced parents' social participation and contacts?, Leisure Studies, 34(4), p. 487-500.

BUisson G., LAPINTE A., 2013, Le couple dans tous ses états. Non-cohabitation, conjoints de même sexe, Pacs..., Insee première, $\mathrm{n}^{\circ} 1435$.

CARR D., 2004, The desire to date and remarry among older widows and widowers, Journal of Marriage and Family, 66(4), p. 1051-1068.

CASSAN F., MAZUY M., ClAnCHÉ F., 2001, Refaire sa vie de couple est plus fréquent pour les hommes, Insee première, $\mathrm{n}^{\circ} 797$.

Castro-Martín T., Domínguez-Folgueras M., Martín-García T., 2008, Not truly partnerless: Non-residential partnerships and retreat from marriage in Spain, Demographic Research, 18(16), p. 443-468.

CHARRIER G., 2008, La mobilité comme aspiration à l'utopie conjugale, in Dervin F., Ljalikova A. (dir.), Regards sur les mondes hypermobiles. Mythes et réalités, Paris, L'Harmattan, Logiques sociales, p. 37-71.

CONnidis I. A., BOREll K., KARLSSON S. G., 2017, Ambivalence and living apart together in later life: A critical research proposal, Journal of Marriage and Family, 79(5), p. 1404-1418.

Coulter R., HU Y., 2017, Living apart together and cohabitation intentions in Great Britain, Journal of Family Issues, 38(12), p. 1701-1729.

Costemalle V., 2015, Parcours conjugaux et familiaux des hommes et des femmes selon les générations et les milieux sociaux, in Insee (collectif), Couples et familles, Edition 2015, Paris, Insee, Insee références, p. 63-76. 
DE JONG GIERVELD J., 2002, The dilemma of repartnering: Considerations of older men and women entering new intimate relationships in later life, Ageing International, 27(4), p. 61-78.

DUNCAN S., PHILliPS M., 2010, People who live apart together (LATs). How different are they?, Sociological Review, 58(1), p. 112-134.

Duncan S., Carter J., Phillips M., Roseneil S., Stoilova M., 2013, Why do people live apart together?, Families, Relationships and Societies, 2(3), p. 323-338.

GIRAUD C., 2017, L’amour réaliste. La nouvelle expérience amoureuse des jeunes femmes, Paris, Armand Colin.

GOLDSCHEIDER F. K., SASSLER S., 2006, Creating stepfamilies: Integrating children into the study of union formation, Journal of Marriage and Family, 68(2), p. 275-291.

HASKEY J., 2005, Living arrangements in contemporary Britain: Having a partner who usually lives elsewhere and living apart together (LAT), Population Trends, $\mathrm{n}^{\circ} 122$, p. $35-45$.

HASKEY J., LEWIS J., 2006, Living-apart-together in Britain: Context and meaning, International Journal of Law in Context, 2(1), p. 37-48.

IVANOVA K., KALMijn M., UUNK W., 2013, The effect of children on men's and women's chances of re-partnering in a European context, European Journal of Population, 29(4), p. 417-444.

LEVIN I., 2004, Living apart together: A new family form, Current Sociology, 52(2), p. 223-240.

Liefbroer A. C., Poortman A.-R., Seltzer J., 2015, Why do intimate partners live apart? Evidence on LAT relationships across Europe, Demographic Research, 32(8), p. 251-286.

MARTIN C., 1994, Diversité des trajectoires post-désunion. Entre le risque de solitude, la défense de son autonomie et la recomposition familiale, Population, 49(6), p. 1557-1583.

PAPON S., BeAumel C., 2018, Bilan démographique 2017. Plus de 67 millions d'habitants en France au $1^{\text {er }}$ janvier 2018, Insee première, $n^{\circ} 1683$.

PASTEElS I., LySSENS-DANNEBOOM V., MORTELMANS D., 2015, A life course perspective on Living Apart Together: Meaning and incidence across Europe, Social Indicators Research, 130(2), p. 799-817.

PRIOUX F., 2009, Les couples non mariés en 2005 : quelles différences avec les couples mariés?, Politiques sociales et familiales, $n^{\circ}$ 96, p. 87-95.

RAPP I., 2018, Partnership formation in young and older age, Journal of Family Issues, 39(13), p. 3363-3390.

RAULT W., RÉGNIER-LOILIER A., 2015, La première vie en couple : évolutions récentes, Population et sociétés, $\mathrm{n}^{\circ} 521$.

RAULT W., RÉGNIER-LOILIER A., 2019, Étudier les parcours individuels et conjugaux en France. Enjeux scientifiques et choix méthodologiques de l'enquête Épic, Population, 74(1-2), p. 11-40.

RÉGNIER-LOILIER A., 2016a, Partnership trajectories of people in stable non-cohabiting relationships in France, Demographic Research, 35(40), p. 1169-1212.

RÉGNIER-LOILIER A., 2016b, Le devenir conjugal des personnes en relation non cohabitante, in Régnier-Loilier A. (dir.), Parcours de familles, Paris, Ined, Grandes enquêtes, p. 111-136. 
RÉGNIER-LOILIER A., 2018, Faire couple à distance : prévalence et caractéristiques, in Imbert C., Lelièvre É., Lessault D., La famille à distance, Paris, Ined, Questions de populations, $\mathrm{n}^{\circ} 2$, p. 165-193.

RÉGNIER-LOILIER A., VignOLi D., 2018, The diverse nature of living apart together relationships. A France-Italy comparison, Journal of Population Research, 35(1), p. 1-22.

RÉGNier-Loilier A., BeAujouAn É., Villeneuve-Gokalp C., 2009, Neither single nor in a couple. A study of living apart together in France, Demographic Research, $\mathrm{n}^{\circ} 21$, p. $75-108$.

REIMONDOS A., EvANS A., GRAY E., 2011, Living-apart-together (LAT) relationships in Australia, Family Matters, n' 87, p. 43-55.

SCHimmele C. M., WU Z., 2016, Repartnering after union dissolution in later life, Journal of Marriage and Family, 78(4), p. 1013-1031.

Singly (DE) F., 2000, Penser autrement la jeunesse, Lien social et Politiques, 43, p. 9-21.

Stewart S. D., MANning W. D., SMOCK P. J., 2003, Union formation among men in the U.S.: Does having prior children matter?, Journal of Marriage and Family, 65(1), p. $90-104$.

Stoilova M., Roseneil S., Crowhurst I., Hellesund T., Santos A. C., 2014, Living apart relationships in contemporary Europe: Accounts of togetherness and apartness, Sociology, 48(6), p. 1075-1091.

Strohm C. Q., Seltzer J. A., Cochran S. D., Mays V. M., 2009, "Living apart together" relationships in the United States, Demographic Research, 21(7), p. 177-214.

TURCOTTE M., 2013, Living apart together, Insights on Canadian Society, March, p. 1-9.

VAnASsche S., CORIJn M., MATthijs K., SwiCEGOOD G., 2015a, Repartnering and childbearing after divorce: Differences according to parental status and custodial arrangements, Population Research and Policy Review, 34(5), p. 761-784.

VANASSCHE S., CORIJN M., MATthiJs K., 2015b, Post-divorce family trajectories of men and women in Flanders, Demographic Research, 32(31), p. 859-872.

Villeneuve-GoKalp C., 1997, Vivre en couple chacun chez soi, Population, 52(5), p. 1059-1081.

VilleneuVE-GOKAlP C., 1994, Après la séparation : conséquences de la rupture et avenir conjugal, in Leridon H., Villeneuve-Gokalp C. (dir.), Constance et inconstances de la famille. Biographies familiales des couples et des enfants, Paris, Ined, Cahier n ${ }^{\circ} 134$, p. 137-164.

Wu Z., SCHIMmele C. M., 2005, Repartnering after first union disruption, Journal of Marriage and Family, 67(1), p. 27-36.

Wu Z., SChimmele C. M., Ouellet N., 2015, Repartnering after widowhood, The Journals of Gerontology - Series B: Psychological Sciences and Social Sciences, 70(3), p. 496-507. 


\section{Arnaud RÉGNIER-LOILIER • NOUVELLE VIE DE COUPLE, NOUVELLE VIE COMMUNE ? PROCESSUS DE REMISE EN COUPLE APRÈS UNE SÉPARATION}

La conjugalité a connu de nombreuses évolutions en France. On assiste, d'une part, à une diversification des formes d'unions (mariage, cohabitation hors mariage, pacs, relations non cohabitantes) et, d'autre part, à la discontinuité des parcours amoureux, marqués par des séparations et remises en couple plus fréquentes. Partant de ce double constat, cet article étudie le lien entre l'histoire conjugale passée et la forme, cohabitation ou non, que prend l'union suivante. La plupart des études sur la remise en couple l'abordent sous le seul prisme de la vie commune, occultant la conjugalité non cohabitante comme forme d'union à part entière et laissant dans l'ombre le processus de remise en couple, plus ou moins long selon les caractéristiques de l'union précédente. Les données de l'enquête Étude des parcours individuels et conjugaux (Épic, Ined-Insee 2013-2014) retracent I'histoire conjugale des répondants en distinguant les périodes de non-cohabitation des périodes cohabitantes. En utilisant des fonctions de survie et des modèles de durée, certains freins à l'emménagement sous le même toit sont identifiés, en particulier pour les femmes, comme la présence d'enfants à charge, le fait d'avoir été marié ou d'avoir vécu une séparation conflictuelle. Un âge avancé à la remise en couple réduit par ailleurs la probabilité de vivre ensemble.

\section{Arnaud Régnier-Loilier • New Partner, New Living ArRangements? The Process of REPARTNERING AFTER SEPARATION}

Conjugality in France has seen a number of changes over recent decades. First, we are witnessing diversified forms of unions beyond the "standard" norm of marriage, such as cohabitation outside marriage, PACS (civil union), and non-cohabiting relationships. Second, intimate relationship trajectories are increasingly marked by discontinuity, with separations and repartnering occurring more frequently. Based on these observations, this article explores the present diversification and discontinuity by looking at the link between past conjugal history and the form taken by the subsequent union, i.e. whether cohabiting or not. Most studies on repartnering approach the subject solely through the prism of living together while overlooking non-cohabiting relationships as a separate form of union, thus shedding no light on the repartnering process, which may take varying amounts of time that depend on the characteristics of the previous union. Using data from the French survey on individual and conjugal trajectories known as EPIC (Étude des parcours individuels et conjugaux, INED-INSEE, 2013-2014), we trace the conjugal histories of the respondents by distinguishing between periods of noncohabitation and cohabitation. Using survival functions and duration models, certain impediments to moving in together are identified-some of which are particularly prominent for women, such as the presence of dependent children, having been married, and having experienced a highly conflictive separation. Increased age at the time of repartnering also reduces the probability of living together.

\section{Arnaud RÉGNIER-LOILIER • ¿NUEVA VIDA DE PAREJA, NUEVA VIDA COMÚN? PROCESO DE RETORNO A LA VIDA DE PAREJA DESPUÉS DE UNA SEPARACIÓN}

La pareja ha conocido numerosas evoluciones en Francia. Asistimos, por un lado, a una diversificación de las formas de unión (matrimonio, cohabitación sin matrimonio, Pacs, relación sin cohabitación) y, por otro lado, a la discontinuidad del recorrido amoroso, caracterizado por separaciones y por retornos a la vida de pareja más frecuentes. A partir de esta doble constatación, este artículo estudia la relación entre la historia de la vida conyugal pasada y la forma, con cohabitación o no, que toma la unión siguiente. La mayoría de los estudios sobre el retorno a la vida de pareja, lo hacen bajo el solo prisma de la vida en común, ocultando así la conyugalidad sin cohabitación como forma de unión plena y dejando en la sombra el proceso de retorno a la vida de pareja, más o menos largo según las características de la unión precedente. Los datos de la encuesta EPIC Estudio de las trayectorias individuales y conyugales (Étude des parcours individuels et conjugaux, InedInsee, 2013-2014) describen la historia conyugal de los entrevistados distinguiendo los periodos de cohabitación de los periodos sin cohabitación. Utilizando funciones de supervivencia y modelos de duración, ciertos frenos a la vuelta a la vida común son identificados, en particular en las mujeres, como la presencia de hijos a cargo, el hecho de haber estado casado o el de haber sufrido una separación conflictiva. Una edad avanzada reduce también la probabilidad de un retorno a la vida común.

Mots-clés : Épic, couple, Living Apart Together, couple non cohabitant, remise en couple, séparation, cohabitation, histoire conjugale, France

Keywords: EPIC, couple, Living Apart Together, non-cohabiting couple, repartnering, separation, cohabitation, conjugal history, France 
